\title{
Rewiring of Afferent Fibers in the Somatosensory Thalamus of Mice Caused by Peripheral Sensory Nerve Transection
}

\author{
Yuichi Takeuchi, ${ }^{1,2,3}$ Miwako Yamasaki, ${ }^{4 \star}$ Yasuyuki Nagumo, ${ }^{1 \star}$ Keiji Imoto,,${ }^{2,3}$ Masahiko Watanabe, ${ }^{4}$ \\ and Mariko Miyata ${ }^{1,5}$ \\ ${ }^{1}$ Department of Physiology, School of Medicine, Tokyo Women's Medical University, Shinjuku-ku, Tokyo 162-8666, Japan, ${ }^{2}$ Department of Physiological Sciences, \\ School of Life Science, The Graduate University for Advanced Studies (SOKENDAI), Okazaki 444-8787, Japan, ${ }^{3}$ Department of Information Physiology, National \\ Institute for Physiological Sciences, Okazaki 444-8787, Japan, ${ }^{4}$ Department of Anatomy, Hokkaido University Graduate School of Medicine, Sapporo 060-8638, \\ Japan, and 5 Precursory Research for Embryonic Science and Technology, Japan Science and Technology Agency, Kawaguchi, Saitama 332-0012, Japan
}

The remodeling of neural circuitry and changes in synaptic efficacy after peripheral sensory nerve injury are considered the basis for functional reorganization in the brain, including changes in receptive fields. However, when or how the remodeling occurs is largely unknown. Here we show the rapid rewiring of afferent fibers in the mature ventral posteromedial thalamic nucleus of mice after transection of the peripheral whisker sensory nerve, using the whole-cell voltage-clamp technique. Transection induced the recruitment of afferent fibers to a thalamic relay neuron within 5- $6 \mathrm{~d}$ of injury. The rewiring was pathway specific, but not sensory experience dependent or peripheral nerve activity dependent. The newly recruited fibers mediated small EPSCs, and postsynaptic GluA2-containing AMPA receptors were selectively upregulated at the new synapses. This rapid and pathway-specific remodeling of thalamic circuitry may be an initial step in the massive axonal reorganization at supraspinal levels, which occurs months or years after peripheral sensory nerve injury.

\section{Introduction}

Peripheral sensory nerve injury results in functional reorganization throughout the spinal cord and brain, such as alteration of the receptive fields. This reorganization closely relates to both the ensuring recovery of the function and the formation of maladaptive neuronal circuitry (Wall et al., 2002; Flor et al., 2006). It is generally thought that structural alterations, including the remodeling of neural circuitry and its synaptic properties, underlie the functional reorganization after peripheral nerve injury (Kaas et al., 2008). Several spinal cord studies have demonstrated that the central axon of $A \beta$ fibers sprouts a few weeks after the peripheral nerve injury (Woolf et al., 1992; Koerber et al., 1994). In contrast, the trans-synaptic remodeling of supraspinal circuitry after peripheral nerve injury still remains poorly understood. Axonal changes at supraspinal levels are believed to take months or even years to take place after peripheral nerve injury. For instance, the decrease in axonal branching of afferent fibers to the primate ventral posterolateral thalamic nucleus (VPL) can be

\footnotetext{
Received 0ct. 2, 2011; revised Feb. 13, 2012; accepted March 11, 2012.

Author contributions: M.M. designed research;Y.T., M.Y., and Y.N. performed research; K.I. and M.W. contributed unpublished reagents/analytic tools; Y.T., M.Y., and Y.N. analyzed data; Y.T., M.Y., Y.N., and M.M. wrote the paper.

This study was supported by Grants-in-Aid for Scientific Research (18500316 and 20021029), the Japan Science and Technology Agency Precursory Research for Embryonic Science and Technology Program, the Smoking Research Foundation, the Naito Science and Engineering Foundation, Takeda Science Foundation, Tokyo Women's Medical University's Institute for Integrated Medical Sciences, and a Grant-in-Aid from the Japan Society for Promotion of Science Fellows. We thank Dr. Y. Katayama and E. Naraba for technical assistance.

${ }^{*} M . Y$. and Y.N. contributed equally to this work.

The authors declare no competing financial interests.

Correspondence should be addressed to Mariko Miyata, Department of Physiology, School of Medicine, Tokyo Women's Medical University, 8-1 Kawada-cho, Shinjuku-ku, Tokyo 162-8666, Japan. E-mail:mmiyata@research.twmu.ac.jp. D0l:10.1523/JNEUROSC1.5008-11.2012

Copyright $\odot 2012$ the authors $\quad 0270-6474 / 12 / 326917-14 \$ 15.00 / 0$
}

seen years after a cuneate fasciculus lesion (Graziano and Jones, 2009), suggesting the retraction of axons and compensatory axonal sprouting from unaffected fibers. In the somatosensory cortex, long-range intracortical axonal sprouting has been reported, but it has also been detected long after amputation (Florence et al., 1998). However, these results are not temporally correlated with the remodeling of cortical spines in a recent study (Kim and Nabekura, 2011), which shows that the spine turnover in the somatosensory cortex markedly increases within a few days after peripheral nerve injury. Thus, the details regarding when and how the remodeling of supraspinal circuitry occurs after peripheral nerve injury are largely unknown. Moreover, the synaptic properties of the remodeled synapses remain to be fully clarified.

Somatosensory information from the maxillary region is conveyed to the ipsilateral trigeminal nuclei via the infraorbital nerve and then to relay neurons in the contralateral ventral posteromedial thalamic nucleus (VPM) via medical lemniscal fibers, afferent fibers of projection neurons in the trigeminal nuclei. Medial lemniscal fibers have unique characteristics in their innervations and synaptic properties. Most relay neurons become innervated by single strong lemniscal fiber after developmental synapse elimination (Arsenault and Zhang, 2006). Such a mature lemniscal fiber induces fast and reliable EPSCs in an all-or-none-fashion, mediated primarily by GluA2-lacking AMPA receptors (AMPARs) (Castro-Alamancos, 2002; Arsenault and Zhang, 2006; Miyata and Imoto, 2006). Therefore, it is very useful to examine lemniscal synaptic properties for detecting changes in innervation pattern and synaptic plasticity at supraspinal levels after peripheral nerve injury.

Here we provide direct evidence that multiple lemniscal fibers were newly recruited to a relay neuron within 1 week after transection of the infraorbital nerve. Moreover, GluA2-containing 
AMPARs were specifically upregulated at postsynaptic sites of newly recruited fibers. This rapid remodeling of thalamic circuitry may be linked to the functional reorganization and massive structural changes in neural circuitry over time after peripheral sensory nerve injury.

\section{Materials and Methods \\ Animals}

All experiments were approved by the Animal Care and Use Committee of the Tokyo Women's Medical University and National Institute for Physiological Sciences, and were performed according to the institutional guidelines concerning the care and handling of experimental animals. C57BL/6NCr mice [postnatal day 21 (P21)-P32] (Nihon SLC, Hamamatsu) of both sexes were used. Mice were provided with a commercial diet and water ad libitum with controlled temperature, humidity, and lightning (12 h light/dark cycle).

\section{Infraorbital nerve cut}

Mice on P21 were anesthetized by intraperitoneal administration of ketamine $(80 \mathrm{mg} / \mathrm{kg})$ and xylazine $(10 \mathrm{mg} / \mathrm{kg})$. The infraorbital nerve on the left side of the face was exposed under a dissecting microscope (see Fig. $1 A)$. The infraorbital nerve was completely transected using sterilized fine scissors, and the cutting planes were separated at a distance of $>1.0$ $\mathrm{mm}$ to prevent regeneration. For sham operation, the infraorbital nerve was exposed but not cut. The skin was then sutured with silken threads. Physiological saline ( $0.2 \mathrm{ml}$ per animal) was subcutaneously injected for preventing dehydration after the surgery.

\section{Whisker deprivation}

All large vibrissae on the left side snout were repeatedly deprived every day from P21 to the recording day. Briefly, mice were anesthetized with isoflurane. Under a dissecting microscope, vibrissae were carefully plucked out using fine tweezers by applying slow, steady tension to the base of the vibrissa until the vibrissa slipped out of the follicle (Li et al., 1995).

\section{Preparation and implantation of EVAFLEX ${ }^{T M}$ pieces}

EVAFLEX $^{\mathrm{TM}}$ (EV40W, DuPont-Mitsui Polychemicals) is the commercial analog of ELVAX ${ }^{\mathrm{TM}}$. Small EVAFLEX ${ }^{\mathrm{TM}}$ pieces $\left(4 \times 2 \times 0.2 \mathrm{~mm}^{3}\right)$ were prepared as previously described (Kakizawa et al., 2005), each containing either saline or $\sim 3 \mathrm{nmol}$ of tetrodotoxin (TTX). For the implantation of EVAFLEX $^{\text {TM }}$ pieces on P21, mice were anesthetized with the ketamine/xylazine mixture and the left infraorbital nerve was exposed. Two EVAFLEX ${ }^{\mathrm{TM}}$ pieces were then transversely placed at both medial and lateral sides of the infraorbital nerve, respectively. The effectiveness of the blockade was assessed in the pilot study by directly recording fiber volleys of the infraorbital nerve (data not shown) (Henderson et al., 1992). For the continuous blockade of the infraorbital nerve, the implants were replaced with new ones on P27, the sixth day after the first implantation.

\section{Slice preparation}

Mice were anesthetized with isoflurane and decapitated. Parasagittal 300$\mu \mathrm{m}$-thick thalamic slices (Arsenault and Zhang, 2006) (see Fig. $1 B$ ) were prepared using a microslicer (VT1200S, Leica) in an ice-cold cutting solution (in mM: 234 sucrose, $2.5 \mathrm{KCl}, 1.25 \mathrm{NaH}_{2} \mathrm{PO}_{4}, 10 \mathrm{MgCl}_{2}, 0.5$ $\mathrm{CaCl}_{2}, 25 \mathrm{NaHCO}_{3}, 0.5$ myo-inositol, and 11 glucose) equilibrated with $95 \% \mathrm{O}_{2}-5 \% \mathrm{CO}_{2}$. Slices were then transferred in an incubation chamber containing artificial CSF (ACSF) (in mM: $125 \mathrm{NaCl}, 2.5 \mathrm{KCl}, 1.25$ $\mathrm{NaH}_{2} \mathrm{PO}_{4}, 1 \mathrm{MgSO}_{4}, 2 \mathrm{CaCl}_{2}, 26 \mathrm{NaHCO}_{3}$, and 20 glucose) equilibrated with $95 \% \mathrm{O}_{2}-5 \% \mathrm{CO}_{2}$. Slices were kept at $32^{\circ} \mathrm{C}$ for $30 \mathrm{~min}$, and then at room temperature until just before the recording. Each slice was transferred into a submerge-type recording chamber and perfused by ACSF kept at $30-32^{\circ} \mathrm{C}$ at a rate of $2.5-3.0 \mathrm{ml} / \mathrm{min}$. During all recordings, $10 \mu \mathrm{M}$ (-)-bicuculline methochrolide and $1 \mu \mathrm{M}$ CGP55845 were added into the superfusate to block $\mathrm{GABA}_{\mathrm{A}}$ and $\mathrm{GABA}_{\mathrm{B}}$ receptors, respectively.

\section{Electrophysiology}

Whole-cell voltage-clamp recordings of lemniscal EPSCs. Patch pipettes were made from borosilicate capillaries (outer diameter, $1.5 \mathrm{~mm}$; inner diameter, $0.87 \mathrm{~mm}$; Hilgenberg). The tip resistance was $2-5 \mathrm{M} \Omega$ when filled with the following intracellular solution (in mM): $120 \mathrm{CsMeSO}_{3}, 10$
HEPES, 1 EGTA, $2 \mathrm{MgCl}_{2}, 0.1 \mathrm{CaCl}_{2}, 20 \mathrm{NaCl}, 5$ QX-314, 2 ATP-Na 2 , and 0.5 GTP-Na, pH 7.3 (290-300 mOsm). Biocytin (0.5\%) was also included for the cell labeling. Liquid junction potential $(+13.0 \mathrm{mV})$ was not compensated unless otherwise noted. Whole-cell voltage-clamp recordings were made from a relay neuron in the VPM or VPL under visual guidance using an upright microscope (BX51WI, Olympus) with an infrared-differential interference contrast (IR-DIC) video system (C3077-79 with C2741-62, Hamamatsu Photonics). The location of the VPM or VPL was validated according to a mouse brain atlas. Recordings and data acquisition were performed using a MultiClamp700A amplifier and Digidata 1322A with pClamp 8 software (MDS) or an EPC9 amplifier with PULSE software (HEKA Elektronik). Signals were filtered at 3 $\mathrm{kHz}(-3 \mathrm{~dB})$ by a four-pole Bessel filter and digitized at $50 \mathrm{kHz}$. Data analysis was performed using Igor Pro software (Wavemetrics). The pipette series resistance (Rs) was compensated by $50-90 \%$. Rs values were monitored throughout the recordings, and recordings were discarded when Rs varied by $>20 \%$. Cells were rejected if Rs increased $>20 \mathrm{M} \Omega$, and only recordings taken under a low Rs condition $(<10 \mathrm{M} \Omega)$ were used in analyses of EPSC kinetics. Experiments were not performed blind.

Lemniscal fiber responses. To evoke lemniscal fiber-mediated EPSCs, a concentric bipolar electrode (tip diameter, $25 \mu \mathrm{m}$; IMB-160820, InterMedical) was used. The stimulus electrode was placed on the medial lemniscal fiber bundle (Arsenault and Zhang, 2006) (see Fig. $1 B$ ). The electrical stimulation consisted of bipolar square pulses (duration, $10 \mu \mathrm{s}$; interval, $100 \mu \mathrm{s}$ ) of constant currents (typically 10-400 $\mu \mathrm{A}$ ), and delivered at $0.1 \mathrm{~Hz}$ via a biphasic isolator (BSI-2, BAK Electronics). We ensured that recorded synaptic currents were activated by lemniscal fibers by following parameters. We routinely used the paired-pulse stimulation with an interstimulus interval of $50 \mathrm{~ms}$ to distinguish lemniscal synaptic responses from corticothalamic responses, because lemniscal responses display depression, whereas corticothalamic responses exhibit facilitation in response to paired stimuli (Castro-Alamancos, 2002; Miyata and Imoto, 2006). In addition, lemniscal EPSCs are elicited in an all-or-none fashion, and they show stepwise increments with the distinct thresholds in response to increasing stimulus intensity (Castro-Alamancos, 2002; Arsenault and Zhang, 2006; Miyata and Imoto, 2006). Moreover, lemniscal EPSCs have a faster rise time than that of corticothalamic EPSCs (typically $<1 \mathrm{~ms}$ ) (Miyata and Imoto, 2006).

Because there was no kainate receptor component in lemniscal EPSCs even after the infraorbital nerve cut (IONC) (data not shown), AMPARand NMDAR-mediated EPSCs were measured as EPSCs recorded at -70 and $+40 \mathrm{mV}$ (>10 $\mathrm{ms}$ after the onset), respectively. The decaying phases of EPSCs at $-90 \mathrm{mV}$ in the presence of $100 \mu \mathrm{M}$ DL-APV and at $+40 \mathrm{mV}$ in the presence of $20 \mu \mathrm{M}$ NBQX were fitted with a single exponential function to determine decay time constants of AMPAR- and NMDARmediated components, respectively (Miyata and Imoto, 2006).

To determine the number of lemniscal inputs for each relay neuron, we recorded evoked lemniscal EPSCs at holding potentials of -70 and $+40 \mathrm{mV}$ from the same cell over a wide range of stimulus intensity (typically from 10 to $400 \mu \mathrm{A}$ ) (Arsenault and Zhang, 2006; Hooks and Chen, 2006, 2008; Wang and Zhang, 2008). First, we roughly searched for EPSC steps using the increment of $50 \mu \mathrm{A}$. After that, we used small increments on the order of $25,10,5$, and $1 \mu \mathrm{A}$ near each transition point to ensure that it was indeed a single step with a stable response. After each accurate threshold was determined, we systematically applied three to five stimuli below and above each threshold at holding potentials of -70 and $+40 \mathrm{mV}$ on the same cell and confirmed that the number of steps was identical in both EPSCs. The IONC induced at most four EPSC steps, all of which were clearly distinguishable. To determine single-fibermediated EPSC amplitude, EPSC responses by three stimuli at each suprathreshold level were averaged. At the end of the counting, we confirmed the absence of extra lemniscal fiber inputs by a strong stimulation, the intensity of which was twofold greater than the largest threshold. Each lemniscal fiber-mediated EPSC was isolated by off-line subtraction. Maximum EPSCs (saturated EPSCs of a given relay neuron) and isolated single lemniscal fiber-mediated EPSCs were subject to offline analyses using Igor Pro.

Cluster analyses. A standard Gaussian mixture model was used for clustering (Fraley and Raftery, 2002; McLachlan and Chang, 2004). Each 


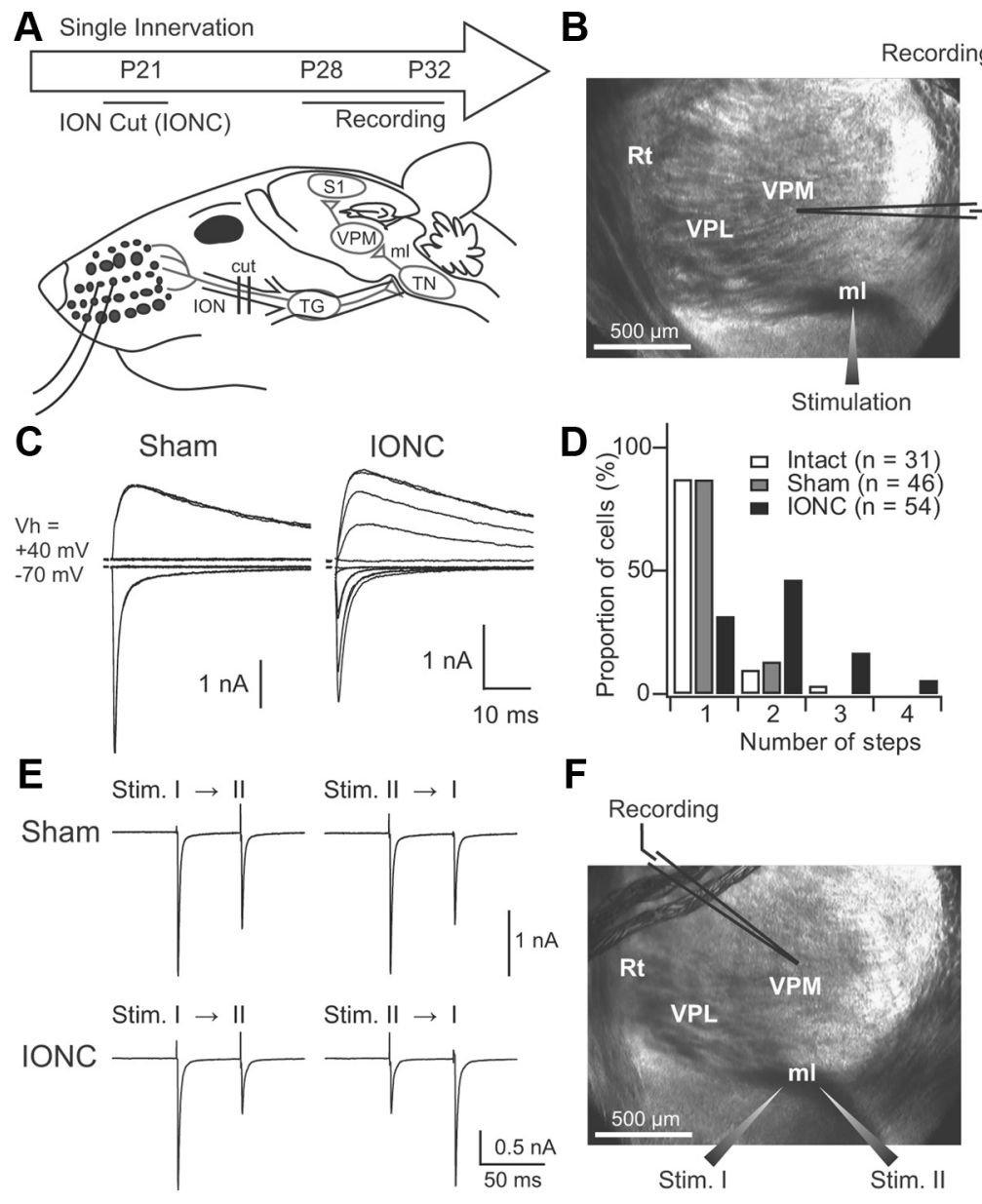

Figure 1. Infraorbital nerve cut-induced multiple innervation of lemniscal fibers onto a relay neuron in the VPM. $A$, The experimental schedule and schematic showing the IONC Operation. $\boldsymbol{B}$, Schematic showing the position of a recorded relay neuron and the stimulation site of medial lemniscal fibers depicted on an infrared IR-DIC image of acute parasagittal thalamic slice of mice. $\boldsymbol{C}$, Representative traces of lemniscal EPSCs recorded 1 week after the sham and the IONC operation under voltage-clamp condition at +40 and -70 $\mathrm{mV}$. Several raw traces with different stimulus intensities are superimposed at each holding potential (Vh). $\boldsymbol{D}$, The distributions of neurons with different numbers of lemniscal inputs for the intact, sham-operated, and IONC-operated VPM. $n$, Number of cells. $\boldsymbol{E}$, Representative traces of lemniscal EPSCs in response to the alternate stimulation (with a 50 ms interstimulus interval) by two electrodes (Stim. I and Stim. II) from the same VPM neuron in the sham and IONC groups. Traces shown are the average of six consecutive EPSCS. $\boldsymbol{F}$, Schematic showing of the position of a recorded relay neuron and stimulation sites on an IR-DIC image of a thalamic slice. $\mathrm{ml}$ Medial lemniscus; Rt, reticular nucleus of thalamus; $\mathrm{S} 1$, primary somatosensory cortex; $\mathrm{TG}$, trigeminal ganglion; $\mathrm{TN}$, trigeminal nuclei.

decay time constant of single-fiber-mediated lemniscal EPSCs at -70 $\mathrm{mV}$ was plotted against its amplitude (see Fig. $4 A-C$ ). The distribution was analyzed by two-dimensional histogram (ordinate bin, $0.2 \mathrm{~ms}$; abscissa bin, $0.2 \mathrm{nA}$ ). Because the histogram of pooled data (from the sham and IONC) exhibited two obvious clusters (see Fig. 4C), the histogram was fitted with the following function, a summation of two independent Gaussian surfaces:

$$
\begin{aligned}
f(x, y)=w_{0} \cdot \exp \left(\frac { - 1 } { 2 ( 1 - w _ { 1 } ^ { 2 } ) } \left(\left(\frac{x-w_{2}}{w_{3}}\right)^{2}+\left(\frac{y-w_{4}}{w_{5}}\right)^{2}\right.\right. & \left.\left.-\frac{2 \cdot w_{1}\left(x-w_{2}\right)\left(y-w_{4}\right)}{w_{3} \cdot w_{5}}\right)\right) \\
+w_{6} \cdot \exp \left(\frac { - 1 } { 2 ( 1 - w _ { 7 } ^ { 2 } ) } \left(\left(\frac{x-w_{8}}{w_{9}}\right)^{2}+\right.\right. & \left(\frac{y-w_{10}}{w_{11}}\right)^{2} \\
& \left.\left.-\frac{2 \cdot w_{7}\left(x-w_{8}\right)\left(y-w_{10}\right)}{w_{9} \cdot w_{11}}\right)\right),
\end{aligned}
$$

where $w_{0}-w_{11}$ are coefficients. Initial guesses of the 12 coefficients were supported by outcomes of the KMeans analysis using an Igor Pro built-in function. The "best" values of the coefficients were then calculated by the Levenberg-Marquardt least-squares method. To find a threshold of
EPSC peak amplitude that well separates the first and second Gaussian surfaces [probability density functions of Weak (W) fibers and Strong (S) fibers in Figure 4C, respectively], we defined two kinds of contamination ratios, Error in $\mathrm{W}$ and Error in S (see Fig. 4D):

Error in W: $f(t)$

$$
=\frac{\text { VUSStrongL }}{\text { VUSWeakL }+ \text { VUSStrongL }}
$$

Error in $S: f(t)$

$$
=\frac{\text { VUSWeakR }}{\text { VUSWeakR + VUSStrongR }}
$$

where four volumes under the surface (VUSs) are functions of sliding threshold ( $t$ : abscissa in Fig. $4 D$ ) as follows:

VUSWeakL: $f(t)$

$$
=\int_{0}^{t} \int_{0}^{\infty} \operatorname{Gaussian}_{1 s t}(x, y) \mathrm{d} x \mathrm{~d} y
$$

VUSWeakR: $f(t)$

$$
=\int_{t}^{\infty} \int_{0}^{\infty} \operatorname{Gaussian}_{1 \mathrm{st}}(x, y) \mathrm{d} x \mathrm{~d} y
$$

VUSStrongL: $f(t)$

$$
=\int_{0}^{t} \int_{0}^{\infty} \operatorname{Gaussian}_{2 \text { nd }}(x, y) \mathrm{d} x \mathrm{~d} y
$$

\section{VUSStrongR: $f(t)$}

$$
=\int_{t}^{\infty} \int_{0}^{\infty} \operatorname{Gaussian}_{2 \mathrm{nd}}(x, y) \mathrm{d} x \mathrm{~d} y,
$$

where the first and second Gaussian surfaces were reproduced using coefficients $\left(w_{0}-w_{11}\right)$ in the Equation 1. In accordance with Equations 2 and 3 , a good threshold at which both Error in W and Error in S were small was searched. That is, the EPSC peak threshold level (in nanoamperes) at which solid and broken lines in Figure $4 D$ cross was explored.

Current-voltage relationships of AMPAR-mediated EPSCs. To preserve intracellular polyamine, which induces an inward rectification of AMPARmediated currents, the pipette solution includes $100 \mu \mathrm{M}$ spermine (Bowie and Mayer, 1995; Isa et al., 1995). Single-fiber-mediated lemniscal EPSCs were recorded in the presence of $200 \mu \mathrm{M}$ D-APV. Current-voltage relationships were investigated by recordings of EPSCs at various holding potentials ranged $\pm 60 \mathrm{mV}$ in $10 \mathrm{mV}$ steps. Liquid junction potential was compensated (estimated as $+13 \mathrm{mV}$ ). The rectification index was defined as the ratio of the EPSC amplitude at $+40 \mathrm{mV}$ to that at $-40 \mathrm{mV}$ (Isa et al., 1995; Wang and Zhang, 2008).

Pharmacological experiments. Single-fiber-mediated lemniscal EPSCs were recorded in the presence of $100 \mu \mathrm{M}$ DL-APV at $-70 \mathrm{mV}$. After establishment of stable recordings, $1 \mathrm{~mm}$ spermine (Isa et al., 1996; Washburn and Dingledine, 1996) or $100 \mu \mathrm{m}$ pentobarbital (Yamakura et al., 1995) was bath applied for $5 \mathrm{~min}$. The average percentage inhibition in amplitude of EPSCs 4-5 min after application was analyzed.

Evoked miniature EPSCs. To analyze input-specific miniature EPSCs, we recorded $\mathrm{Sr}^{2+}$-induced delayed miniature EPSCs (Wang and Zhang, 2008). First, single-fiber-mediated lemniscal EPSCs were recorded in the 

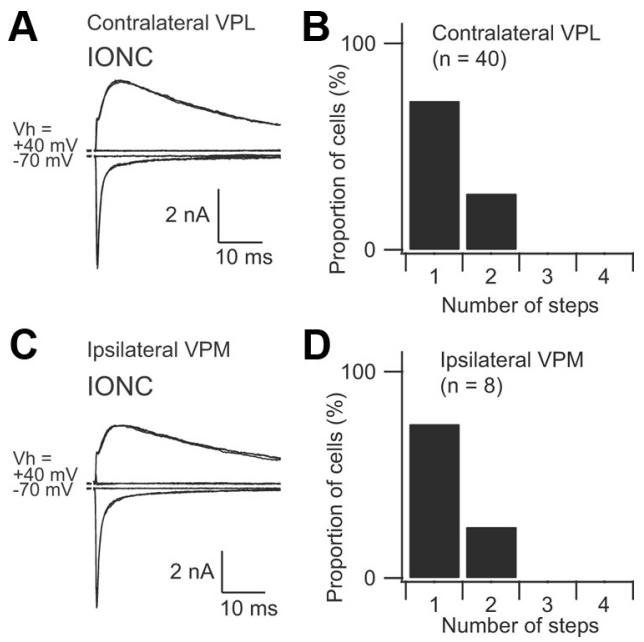

Figure 2. IONC did not induce multiple-innervation of lemniscal fibers in the contralateral VPL or the ipsilateral VPM. The left infraorbital nerve was cut on P21. $A-D$, Lemniscal EPSCs were recorded from relay neurons in the contralateral (right) VPL $(\boldsymbol{A}, \boldsymbol{B})$ and in the ipsilateral (left) VPM $(\boldsymbol{C}, \boldsymbol{D})$, respectively, on P28-P32.A, Representative traces of lemniscal EPSCs in the contralateral VPL showing an all-or-none fashion. $\boldsymbol{B}$, The distributions of neurons in the contralateral VPL with different numbers oflemniscal inputs. $n$, Number of cells. $\boldsymbol{C}, \boldsymbol{D}$, The same as in $\boldsymbol{A}$ and $\boldsymbol{B}$, but in the ipsilateral VPM.

presence of $100 \mu \mathrm{M}$ DL-APV. Slices were then perfused by modified ACSF substituting $4 \mathrm{~mm} \mathrm{Sr}^{2+}$ ions for $\mathrm{Ca}^{2+}$ ions. During the switch of solutions, test stimulations were delivered at $0.1 \mathrm{~Hz}$ and the waveform of EPSCs was continuously monitored to ensure that the same single fiber was stimulated. The holding potential was set at $-90 \mathrm{mV}$ to increase the driving force. A time window for analysis was 100-500 ms after each stimulus. Miniature EPSCs were detected and analyzed by a semiautomated Igor Pro procedure.

Current-clamp recordings. Current-clamp recordings from relay neurons in the VPM were obtained as previously described (Miyata and Imoto, 2006), using an EPC10 amplifier with PATCHMASTER software (HEKA Elektronik). The concentration of potassium chloride in the ACSF was elevated to $3.5 \mathrm{~mm}$ to mimic the endogenous resting potential when the whole-cell configuration was made. Patch pipettes were filled with an internal solution containing the following (in $\mathrm{mM}$ ): 138 K-gluconate, $4 \mathrm{KCl}, 2 \mathrm{NaCl}, 10 \mathrm{HEPES}, 0.2$ EGTA, 4 ATP-Mg, $0.3 \mathrm{GTP}-$ Tris, and 7 phosphocreatine-di(Tris), adjusted to $\mathrm{pH} 7.25$ with $\mathrm{KOH}$. Liquid junction potential $(+14.7 \mathrm{mV})$ was compensated.

\section{Immunohistochemistry}

Under deep pentobarbital anesthesia (100 mg/kg body weight, i.p.), IONC-operated mice were fixed transcardially with $4 \%$ paraformaldehyde in $0.1 \mathrm{~m}$ sodium PB, pH 7.2, dehydrated in graded alcohols, and embedded in paraffin blocks. Coronal sections were made using a sliding microtome (4 $\mu \mathrm{m}$ in thickness; SM1000R; Leica Microsystems) and mounted on silane-coated glass slides. Sections were first subjected to pepsin pretreatment for antigen exposure (i.e., incubation in $1 \mathrm{mg} / \mathrm{ml}$ pepsin) (DAKO) in $0.2 \mathrm{~N} \mathrm{HCl}$ for $10 \mathrm{~min}$ at $37^{\circ} \mathrm{C}$. The following immunohistochemical procedures were performed at room temperature $\left(25^{\circ} \mathrm{C}\right)$ : sections to be shown at low magnifications (see Fig. $8 \mathrm{~A}$ ) were incubated successively with $10 \%$ normal goat serum (Nichirei) for 20 min, rabbit anti-GluA2 (1 $\mu \mathrm{g} / \mathrm{ml})$ (Yamazaki et al., 2010) overnight, biotinylated goat anti-rabbit IgG (Nichirei) for $2 \mathrm{~h}$, and avidin-biotinperoxidase complex (Nichirei) for $1 \mathrm{~h}$; and were visualized using the tyramide signal amplification-Cy3 system (PerkinElmer). For quantification of GluA2 immunofluorescent signals, sections were incubated successively with $10 \%$ normal donkey serum (Jackson ImmunoResearch) for $20 \mathrm{~min}$, and a mixture of rabbit anti-GluA2 $(2 \mu \mathrm{g} / \mathrm{ml})$ and guinea pig anti-type vesicular glutamate transporter (VGluT2; $1 \mu \mathrm{g} / \mathrm{ml}$ ) (Miyazaki et al., 2003) overnight; and then incubated with Alexa Fluor 488- and Cy3-conjugated species-specific secondary antibodies for $2 \mathrm{~h}$ (Invitrogen and Jackson ImmunoResearch). Images were taken with a confocal laser scanning microscope (FV1000; Olympus). Photomon- tage covering the ventrobasal complex was assembled from lowmagnification confocal images using MetaMorph software (Molecular Devices). To quantitate GluA2-expressing levels, single optical sections were acquired using identical acquisition settings with a $60 \times$ Plan PlanApoN objective lens $(60 \times / 1.42$, oil-immersion; Olympus) with a $3 \times$ digital zoom. After converting separate color components into the grayscale, the fluorescence intensity (arbitrary units) of each ROI placed on VGluT2-positive terminals was measured by MetaMorph. Data from three mice were pooled together, because there was no significant difference in the scores from three animals examined. Statistical significance was evaluated by Mann-Whitney $U$ test.

\section{Morphological analysis of relay neurons}

Double staining of cytochrome oxidase and biocytin was conducted to analyze morphology of the recorded relay neuron and to confirm that the relay neuron was in the intended thalamic nucleus (Wiser and Callaway, 1996). Completely reconstructed relay neurons were used for morphological analyses of each group. Projection images of relay neurons were drawn using a camera lucida. Pictures were digitized by a scanner, and then binarized and analyzed (dendritic area and soma area) using ImageJ software (version 1.42, http://rsbweb.nih.gov/ij/).

\section{Statistics}

All values were given as means \pm SEM unless otherwise noted. Electrophysiological data analyses, fitting, and cluster analyses were performed by Igor Pro. Several statistical tests were used in accordance with the experimental designs (for details, see Results and figure legends). The significance level was set at $p<0.05$. SPSS (SPSS), EXCEL Statistics (ESUMI), or Igor Pro software was used for statistical analyses.

\section{Drugs}

D-APV, DL-APV, NBQX, (-)-bicuculline methochrolide, and CGP55845 were purchased from Tocris Cookson. Pentobarbital sodium was purchased from Dainippon Sumitomo Pharma. TTX was purchased from Wako Pure Chemical Industries. Ketamine hydrochloride was purchased from Daiichi Sankyo. Spermine and xylazine hydrochloride were purchased from Sigma-Aldrich. Isoflurane was purchased from Abbott Japan. EVAFLEX ${ }^{\mathrm{TM}}$ (EV40W) was a gift from DuPont-Mitsui Polychemicals. All other chemicals were purchased from Sigma-Aldrich, Wako Pure Chemical Industries, Nacalai Tesque, or Kanto Chemical.

\section{Results}

\section{Multiple innervation of lemniscal fibers onto a relay neuron after the infraorbital nerve cut}

We studied postoperative changes at synapses between lemniscal fibers and a VPM relay neuron (lemniscal synapses) on parasagittal brain slices after transection of the infraorbital nerve, which is the primary sensory nerve from vibrissae follicle receptors to the brainstem. Most VPM relay neurons are innervated by only one or two lemniscal fibers after redundant lemniscal synapses are developmentally eliminated by P17 (Arsenault and Zhang, 2006). Thus, we applied an IONC on the left side of the face on P21 (Fig. 1A), after the synaptic elimination process. We performed whole-cell patch-clamp recordings from relay neurons located in the contralateral VPM on postoperative day 7 (POD 7)-POD11 (P28-P32) (Fig. 1A,B). The lemniscal fiber bundle was visually identified and electrical stimulation to the bundle reliably elicited lemniscal EPSCs in VPM relay neurons (Fig. $1 B, C)$. One week after the IONC, we assessed the number of lemniscal fiber inputs to a single relay neuron by step-counting procedures, as previously described (Arsenault and Zhang, 2006; Wang and Zhang, 2008). We counted the number of EPSC steps with an increase of stimulus intensity (see Materials and Methods).

During this postmaturation period (P28-P32), we observed that most relay neurons in the intact and sham groups were innervated by a single lemniscal fiber (mono-innervation); the neu- 
A

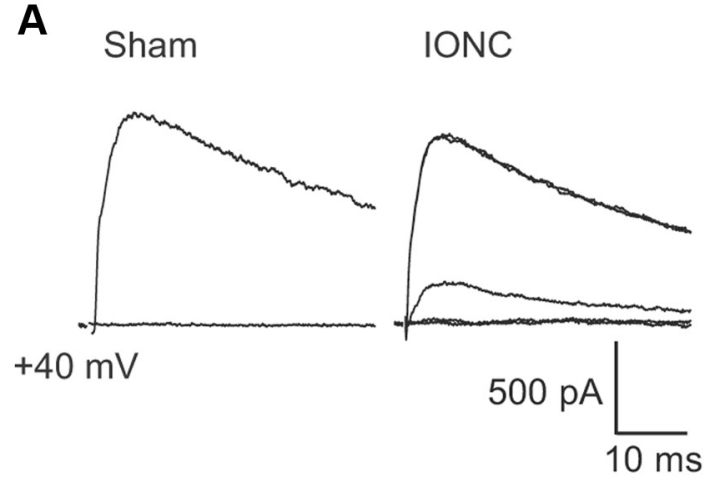

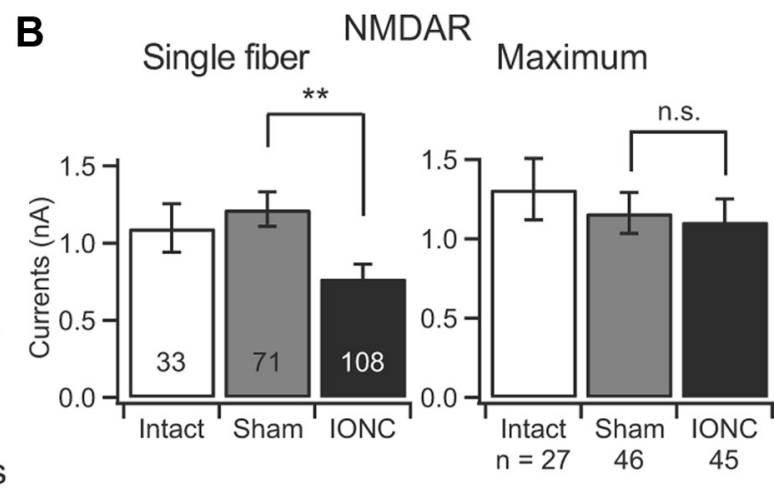
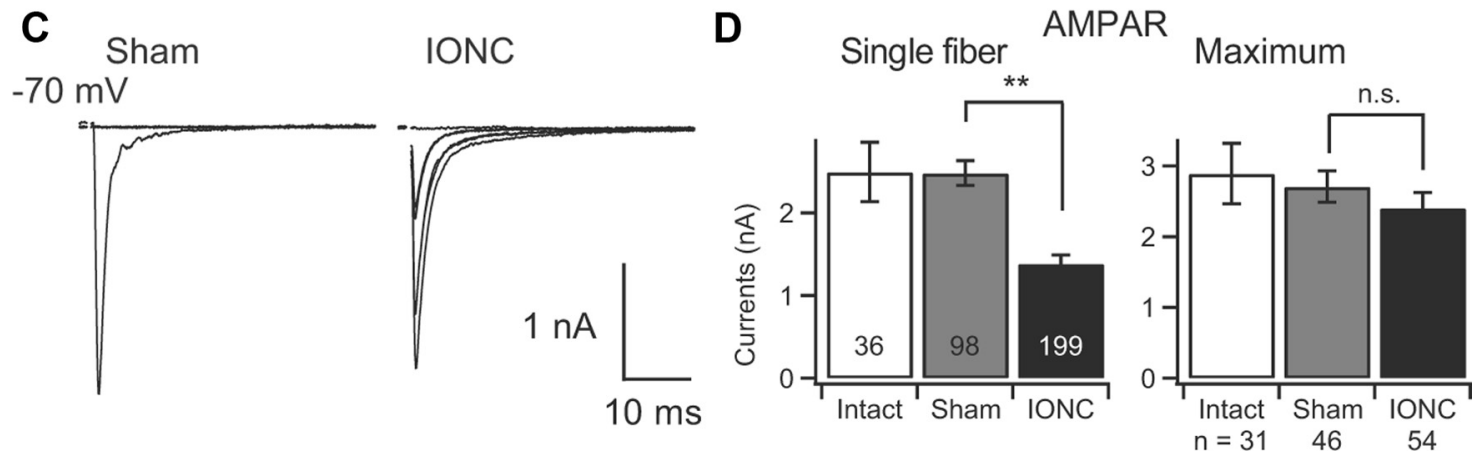

Figure 3. IONC reduces the mean amplitude of single-fiber-mediated lemniscal EPSCS. $A, C$, Representative traces of lemniscal EPSC from both the sham-and IONC-operated mice are shown with the same scale. Several raw traces with different stimulus intensities are superimposed. Holding potentials were $+40 \mathrm{mV}(\boldsymbol{A})$ and $-70 \mathrm{mV}(\boldsymbol{C})$ for NMDAR-and AMPAR-mediated EPSCS, respectively. Traces shown in $\boldsymbol{A}$ and $\boldsymbol{C}$ were recorded from different relay neurons. $\boldsymbol{B}, \boldsymbol{D}$, Summary bar graphs showing the mean amplitude of single-fiber-mediated (left) and maximal (right) lemniscal EPSCs. Each bar represents the mean \pm SEM. Numbers in columns mean numbers of fibers. $n$, Number of cells. Statistical significance was tested using two-tailed Student's $t$ test. ${ }^{* *} p<0.01$. $n$.S., Not significant.

rons showed a single step in the synaptic response, all of which exhibited an all-or-none response [27/31 recorded neurons in the intact group $(87.1 \%) ; 40 / 46$ recorded neurons in the sham group $(87.0 \%)$ ]. Surprisingly, $68.5 \%(37 / 54)$ of relay neurons in the IONC group showed two or more steps (at most four steps), all of which were clearly distinguishable, and each EPSC was stable. Only $31.5 \%$ (17/54) showed a single step (Fig. $1 D$ ) in the IONC group. Overall, 12 of 54 relay neurons $(22.2 \%)$ in the IONC group showed more than three steps. Such a high degree of EPSC steps was quite unusual in both the intact and sham groups $[p<0.001$, IONC vs sham, two-tailed Kolmogorov-Smirnov (KS) two-sample test]. These results strongly suggest that the IONC recruited additional lemniscal fibers onto a single VPM neuron (multiple innervation). To ensure that different fibers innervated a VPM neuron after the IONC, we tested the fiber independency by paired-pulse effects with two stimulus electrodes (Dunwiddie and Lynch, 1978). Two independent stimulus electrodes (Stim I and Stim II), separated by a distance of $>100$ $\mu \mathrm{m}$, were placed on the lemniscal fiber bundle (Fig. $1 F$ ). We first confirmed that each stimulation reliably elicited lemniscal EPSCs that displayed the paired-pulse depression by the paired stimuli with a $50 \mathrm{~ms}$ interstimulus interval from the same VPM neuron. Next, lemniscal EPSCs were elicited by alternate stimulation using Stim I and Stim II with a $50 \mathrm{~ms}$ interstimulus interval. If the two EPSCs originate from two independent lemniscal fibers, these EPSCs amplitudes should never interfere with each other and should exhibit no paired-pulse depression. Indeed, in 6 of 16 neurons in the IONC group, the stimulation of Stim I and Stim II elicited distinct EPSCs, and these EPSC amplitudes did not interfere with each other by the alternate stimulation (i.e., these EPSCs exhibited no paired-pulse depression by the alternate stimula- tion) (Fig. $1 E$, bottom). This result provides strong evidence that a VPM neuron was truly innervated by different lemniscal fibers in a subset of neurons in the IONC group. In contrast, in the sham group, we never observed such responses in all of the recorded neurons ( $n=16$ cells) (Fig. $1 E$, top). All of the pairs of EPSCs have the same amplitude and exhibited the paired-pulse depression by the alternate stimulation, indicative of monoinnervation in the sham group ( $\operatorname{cham}$ vs IONC, $p<0.05, \chi^{2}$ test for independence).

In contrast to the right VPM, we found that most neurons in the right VPL (the body-part subdivision in the ventrobasal complex) $(72.5 \%, 29 / 40)$ and left VPM (the ipsilateral side of the VPM; 75.0\%, 6/8) still showed mono-innervation after the IONC (Fig. 2). Thus, the IONC-induced rewiring by multiple lemniscal fibers was a pathway-specific change, which occurred in the VPM contralateral to the transected infraorbital nerve.

\section{IONC reduces amplitudes of single-fiber-mediated lemniscal EPSCs}

The amplitudes of NMDAR- and AMPAR-mediated components of individual and maximal lemniscal EPSCs were compared between the sham and IONC groups. The mean peak amplitudes of both AMPAR- and NMDAR-mediated EPSCs of individual lemniscal fibers in the IONC group were significantly smaller than those in the sham group (Fig. 3A,C) [AMPARmediated EPSCs: $1.39 \pm 0.11 \mathrm{nA}(n=199$ fibers $)$ and $2.49 \pm 0.15$ $\mathrm{nA}(n=98$ fibers), respectively, for IONC and sham groups (Fig. $3 C, D$, left; ${ }^{* *} p<0.01, t$ test); NMDAR-mediated EPSCs: $0.77 \pm$ $0.09 \mathrm{nA}(n=108$ fibers $)$ and $1.22 \pm 0.11 \mathrm{nA}(n=71)$, respectively, for IONC and sham groups (Fig. $3 A, B$, left; ${ }^{* *} p<0.01$ )]. However, the mean peak amplitudes of maximal lemniscal 
EPSCs of both AMPAR- and NMDARmediated components were not different [maximal AMPAR-mediated EPSCs: $2.41 \pm 0.22 \mathrm{nA}(n=46$ cells $)$ and $2.71 \pm$ $0.22 \mathrm{nA}(n=54)$, respectively, for IONC and sham (Fig. $3 C, D$, right; $p=0.32$ ); maximal NMDAR-mediated EPSCs: $1.12 \pm 0.15 \mathrm{nA}(n=45$ cells $)$ and $1.16 \pm$ $0.13(n=46)$, respectively, for IONC and sham (Fig. $3 A, B$, right; $p=0.83$ )]. These results indicate that, although lemniscal EPSCs of individual fibers were weakened after the IONC, the compensatory innervation of lemniscal fibers maintained maximal lemniscal EPSCs in a relay neuron.

Weak and strong fibers after the IONC To further understand the synaptic properties of the newly recruited fibers after the IONC, lemniscal fibers were classified by the correlation between decay time constants and amplitudes of AMPARmediated EPSCs for individual lemniscal fibers, using a standard Gaussian mixture model-based technique (Fraley and Raftery, 2002; McLachlan and Chang, 2004). In brief, we first plotted a twodimensional distribution between decay time constants and amplitudes, and the distribution was then fitted with a summation of two independent Gaussians (see Materials and Methods) (Fig. 4A-C). All recorded lemniscal EPSCs in the sham and IONC groups (POD7-POD11) were pooled together and separated into two clusters, which could be well discriminated by the $500 \mathrm{pA}$ threshold of EPSC peak amplitude with negligible contamination (Fig. 4D). This way, we classified the two clusters into $\mathrm{W}$ - and S-fiber groups. In the $\mathrm{W}$-fiber group, peak EPSC amplitudes were typically $<500 \mathrm{pA}(0.24 \pm 0.02 \mathrm{nA}, n=65$ fibers). These amplitudes were evidently smaller than those in sham lemniscal EPSCs. In the S-fiber group, in contrast, EPSCs typically exhibited $>500$ pA for peak EPSC amplitude $[2.37 \pm 0.13 \mathrm{nA}(n=90$ fibers $)$ in the sham group, and $2.06 \pm$ $0.13 \mathrm{nA}$ ( $n=114$ fibers) in the IONC group]. Interestingly, we found that fibers in the IONC group consisted of both $\mathrm{W}$ - and $\mathrm{S}$-fibers, whereas fibers in the sham group consisted exclusively of S-fibers.

In addition, the mean decay time constant of W-fiber EPSCs ( $2.84 \pm 0.18 \mathrm{~ms}, n=46$ fibers) was significantly larger than that of S-fibers in the sham group (sham fibers: $1.68 \pm 0.12 \mathrm{~ms}, n=$ 49 ) and S-fibers in the IONC group ( $1.94 \pm 0.09 \mathrm{~ms}, n=46)$ (Fig. $4 E ; p<0.001$, two-tailed multiple $t$ test with Bonferroni's correction following one-way ANOVA). However, there was no significant difference in $10-90 \%$ rise time of EPSCs between them [Fig. $4 E ; p=0.83$, one-way ANOVA, values: $0.5 \pm 0.0(n=47)$, $0.5 \pm 0.0(n=46)$, and $0.5 \pm 0.0 \mathrm{~ms}(n=46)$, respectively, for sham fibers, S-fibers in the IONC group, and W-fibers in the IONC group]. Because the dendrite branching, soma size, and basic membrane properties of recorded neurons were not respectively].
B
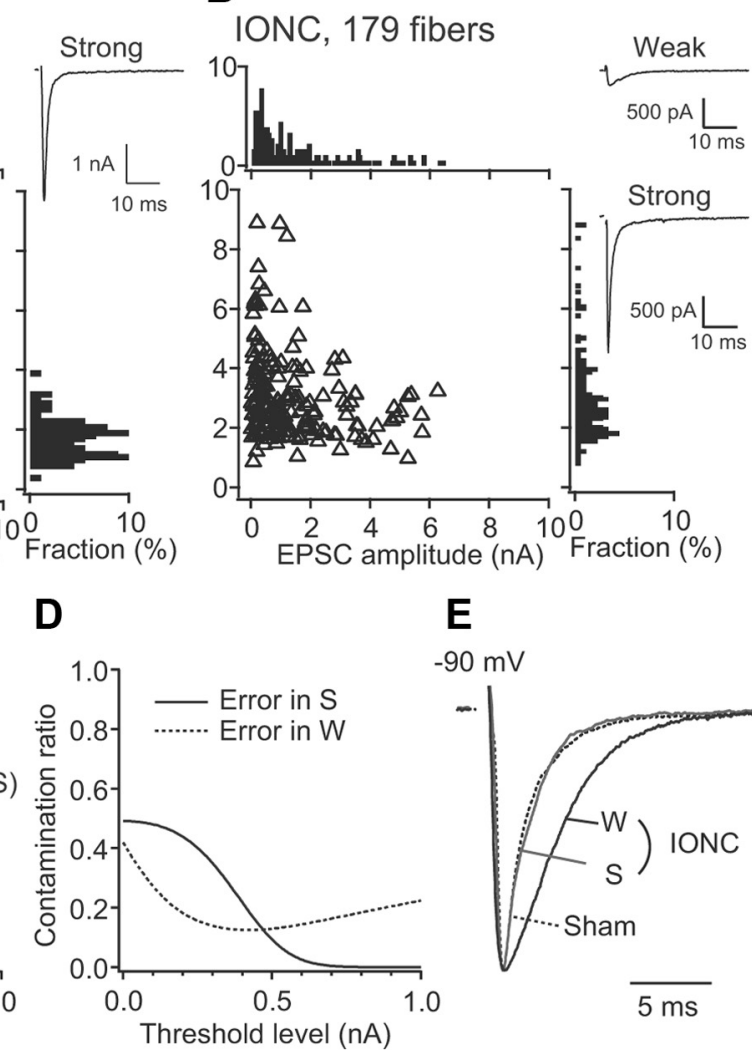

Figure 4. Single-fiber-mediated lemniscal EPSCs after the IONC are classified into weak and strong fiber groups. $\boldsymbol{A}$, Decay time constant of single-fiber-mediated lemniscal EPSCs is plotted against its peak amplitude. Each symbol represents data independent Gaussian surfaces (see Materials and Methods). The two Gaussian surfaces represent two separable clusters PAR component was isolated in $100 \mu \mathrm{m}$ DL-APV at $-90 \mathrm{mV}$. Traces shown are normalized in amplitude $[2633,1436$, and 551 pA for a sham fiber (broken line), an S-fiber in the IONC group (gray line), and a W-fiber in the IONC group (black line),

changed by the IONC operation [sham and IONC, respectively: number of primary dendrites: $6.8 \pm 0.8$ and 7.2 \pm 0.5 (both $n=$ 6 cells); dendritic area: $3174 \pm 311$ and $3225 \pm 314 \mu \mathrm{m}^{2}$ (both $n=6$ cells); soma area: $175 \pm 20$ and $192 \pm 23 \mu \mathrm{m}^{2}$ (both $n=6$ cells); resting membrane potential: $-75.1 \pm 0.8 \mathrm{mV}(n=16$ cells $)$ and $-74.5 \pm 1.0 \mathrm{mV}(n=17$ cells $)$; input resistance: $112.1 \pm 10.6 \mathrm{M} \Omega(n=16$ cells $)$ and $111.6 \pm 12.7 \mathrm{M} \Omega(n=17$ cells); membrane capacitance: $82.9 \pm 2.6$ and $76.8 \pm 3.7 \mathrm{pF}$ (both $n=26$ cells) ( $p>0.05$ for all, unpaired $t$ test], the larger decay time constant of W-fiber EPSCs in the IONC group was unlikely due to the dendritic filtering from the soma to the fibers' innervation sites.

\section{Time course of rewiring after the IONC}

When did the rewiring of lemniscal fibers occur, and which fiber type (W- or S-fiber) was newly recruited? To address these questions, we investigated postoperative changes of innervation by $\mathrm{W}$ - and S-fibers onto a relay neuron. Before the IONC, most relay neurons (on P21) were innervated by a single S-fiber (S-type: $82.5 \%$; 33/40 recorded neurons), while others were innervated by two S-fibers (SS-type: 17.5\%; 7/40) 


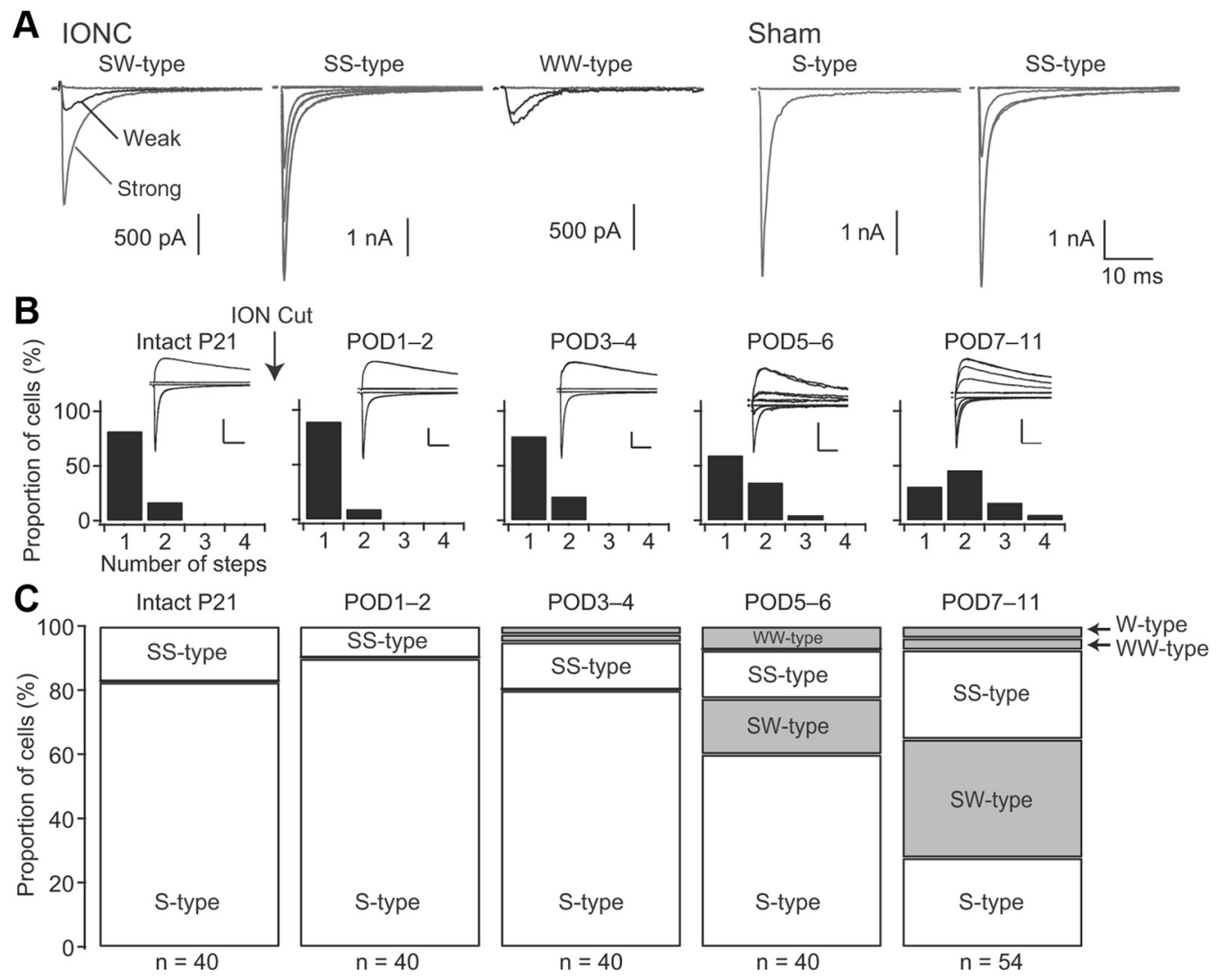

Figure 5. Time course of the rewiring after the IONC. $A$, Various innervation patterns of lemniscal fibers observed on POD7-POD11. S- and W-fiber-mediated EPSCS in the IONC group are represented as gray and black lines, respectively. Several raw traces at $-70 \mathrm{mV}$ with different stimulus intensities are superimposed. The $S$-type receives innervation from a single $S$-fiber; $S S$ - and WW- types indicate relay neurons innervated by multiple S- and W-fibers, respectively; the SW-type is innervated by both S-and W-fibers. $\boldsymbol{B}$, The distributions of neurons with different numbers of lemniscal inputs showing the time course of the rewiring after the IONC. Arrow indicates the operation day, P21. The first graph represents the data from intact P21 mice and the remaining four graphs from the IONC mice of POD1-POD2, POD3-POD4, POD5-POD6, and POD7-POD11, respectively. Insets show representative voltage-clamp traces of lemniscal EPSCs from each recording day. The holding potential was -70 or $+40 \mathrm{mV}$. All time scales: $10 \mathrm{~ms}$. Current scales: 2, 2, 2, 0.5, and $1 \mathrm{nA}$ for P21, P0D1-P0D2, P0D3-P0D4, P0D5-P0D6, and P0D7-P0D11, respectively. C, Progressive changes in the way of lemniscal fibers innervating relay neurons on P21 and subsequent PODs. Shaded regions indicate the portions of relay neurons that contain input from W-fibers. Numbers of recorded cells are given at the bottom.

(Fig. $5 A, C$ ). After the IONC, however, the proportion of S-type neurons markedly decreased around POD5-POD6 $(60 \% ; 24 / 40)$, dropping to a proportion of $27.8 \%$ on POD7POD 11 (15/54). In contrast, the neurons that were innervated by an S-fiber and one or more W-fibers (SW-type) became evident on POD5-POD6 (17.5\%; 7/40), and by POD7-POD11 they were the most common neuron type (37.0\%; 20/54). We also found a small number of neurons that were innervated by a single $\mathrm{W}$-fiber (W-type) or a few W-fibers (WW-type). Overall, the proportion of neurons containing $\mathrm{W}$-fibers (SW-, WW-, and W-types) became statistically significant on POD5-POD6 compared with the proportion in intact mice on P21 $(p<0.01)$ (Fig. 5C; two-tailed multiple $z$-test with Bonferroni's correction following $\chi^{2}$ test for independence). It is noteworthy that most relay neurons $(>90 \%)$ in the IONC group received at least one $S$-fiber over the postoperative period (SW-, SS-, and S-types) (Fig. 5C). Furthermore, the number of neurons innervated by multiple lemniscal fibers also became significant on POD6 ( $p<0.05$ ) (Fig. 5B; two-tailed Steel test following Kruskal-Wallis test, $n=40$ and 20 cells for P21 and each of the postoperative days, respectively). These results indicate that, putatively, $\mathrm{W}$-fibers were newly recruited lemniscal fibers and the new innervation occurred around POD5-POD6. S-fibers were presumably pre-existing ones.

\section{Miniature EPSCs of lemniscal fibers in the sham and IONC groups}

The differences in the amplitude and decay time of AMPARmediated EPSCs between W- and S-fibers may result from altered quantal events at those synapses. To examine this possibility, we analyzed the kinetics of asynchronous miniature EPSCs of individual lemniscal fibers in three fiber groups ( $\mathrm{S}$ - and $\mathrm{W}$-fibers in the IONC group, and sham fibers) by replacing $2 \mathrm{mM} \mathrm{Ca}^{2+}$ with $4 \mathrm{mM} \mathrm{Sr}^{2+}$ in the ACSF with $100 \mu \mathrm{M}$ DL-APV, a specific NMDAR antagonist, on POD7-POD11.

Stimulation of individual lemniscal fibers robustly elicited asynchronous miniature EPSCs under $\mathrm{Sr}^{2+}$-containing ACSF (Fig. 6A). The mean amplitudes of miniature EPSCs from both $\mathrm{S}$ fibers $(18.8 \pm 1.2 \mathrm{pA}, p<0.001)$ and $\mathrm{W}$-fibers $(16.9 \pm 1.1 \mathrm{pA}$, $p<0.001)$ in the IONC group were significantly smaller than those from sham fibers $(28.5 \pm 1.9 \mathrm{pA}$ ) (Fig. $6 \mathrm{~B}, \mathrm{C}$; two-tailed multiple $t$ test with Bonferroni's correction following one-way ANOVA), indicating that the number of individual postsynaptic AMPARs decreased at lemniscal fiber synapses after the IONC. Interestingly, the mean decay time constant of miniature EPSCs from $\mathrm{W}$-fibers $(2.64 \pm 0.19 \mathrm{~ms})$ was significantly larger than that from sham fibers $(1.63 \pm 0.14 \mathrm{~ms}, p<0.001)$ and that from $S$-fibers in the IONC group $(2.03 \pm 0.10 \mathrm{~ms}, p<0.05)$ (Fig. $6 D, E)$, whereas the mean $10-90 \%$ rise time of the three fiber 
groups were virtually identical (Fig. $6 D, F$; $p=0.96$, one-way ANOVA; $0.6 \pm 0.0$, $0.6 \pm 0.0$, and $0.6 \pm 0.0 \mathrm{~ms}$, respectively, for lemniscal EPSCs in sham fibers, $\mathrm{S}$-fibers in the IONC group, and W-fibers in the IONC group).

\section{Relatively abundant GluA2-containing AMPARs at W-fiber synapses}

The kinetics of AMPAR-mediated currents largely depends on their subunit compositions (Mosbacher et al., 1994; Liu and Zukin, 2007). Thus, switches of AMPAR compositions may account for the prolonged decay time of miniature EPSCs and evoked lemniscal EPSCs at W-fiber synapses after the IONC. Because GluA2containing AMPARs have slower kinetics than do GluA2-lacking AMPARs (Geiger et al., 1995; Fuchs et al., 2001), one possibility is that GluA2-containing AMPARs are upregulated at synapses of $\mathrm{W}$-fibers. Thus, we first investigated the currentvoltage relationship $(I-V)$ of AMPARmediated EPSCs evoked by stimulating individual lemniscal fibers on POD7POD11 (Fig. 7A-C) in the presence of 200 $\mu \mathrm{M}$ D-APV. AMPAR-mediated EPSCs of sham fibers and S-fibers in the IONC group exhibited a strong inward rectification (Fig. $7 A, B)(n=11$ and 14 fibers, respectively, for sham fibers and S-fibers in the IONC group), suggesting that they were mainly mediated by GluA2-lacking subunit compositions as well as normal lemniscal EPSCs (Arsenault and Zhang, 2006; Wang and Zhang, 2008). However, EPSCs elicited by stimulation of a W-fiber $(n=10)$ exhibited a significantly weak inward rectification compared with those of sham fibers and S-fibers in the IONC group (Fig. $7 A, B$, both $p<0.01$, two-way repeated ANOVA) (Fig. $7 B$; W-fiber vs sham fiber, ${ }^{\star} p<0.05$, ${ }^{* *} p<0.01$, two-tailed multiple $t$ test with Bonferroni's correction following one-way ANOVA). The mean rectification index (EPSCs at $+40 \mathrm{mV} /$ at $-40 \mathrm{mV})$ of $\mathrm{W}$-fibers $(0.44 \pm 0.05)$ was significantly larger than those of sham fibers $\left(0.29 \pm 0.03,{ }^{*} p<\right.$ $0.05)$ and S-fibers in the IONC group $\left(0.26 \pm 0.02,{ }^{* *} p<0.01\right)$ (Fig. 7C; two-tailed multiple $t$ test with Bonferroni's correction following one-way ANOVA). Next, we conducted pharmacological experiments using spermine and pentobarbital, specific inhibitors for GluA2-lacking and GluA2-containing AMPARs, respectively (Yamakura et al., 1995; Isa et al., 1996; Washburn and Dingledine, 1996). We found that bath-applied $1 \mathrm{~mm}$ spermine was less effective for inhibiting EPSCs of W-fibers (37.0 \pm $5.6 \%, n=8$ fibers) than it was for inhibiting EPSCs of sham fibers $\left(57.7 \pm 3.6 \%,{ }^{* *} p<0.01, n=7\right)$ and S-fibers in the IONC group $\left(63.0 \pm 2.9 \%,{ }^{* *} p<0.001, n=11\right)$ (Fig. $\left.7 D, E\right)$. In contrast, bath-applied $100 \mu \mathrm{M}$ pentobarbital inhibited EPSCs of W-fibers (30.0 $\pm 11.1 \%, n=7$ fibers) more efficiently than those of sham fibers $\left(8.3 \pm 1.8 \%,{ }^{\star} p<0.05, n=10\right)$ and S-fibers in the IONC group $\left(9.2 \pm 2.4 \%,{ }^{\star} p<0.05, n=7\right)$ (Fig. $\left.7 F, G\right)$. These results indicate that GluA2-containing AMPARs were relatively abundant at W-fiber synapses.

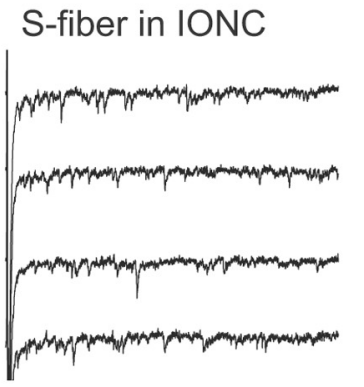

W-fiber in IONC
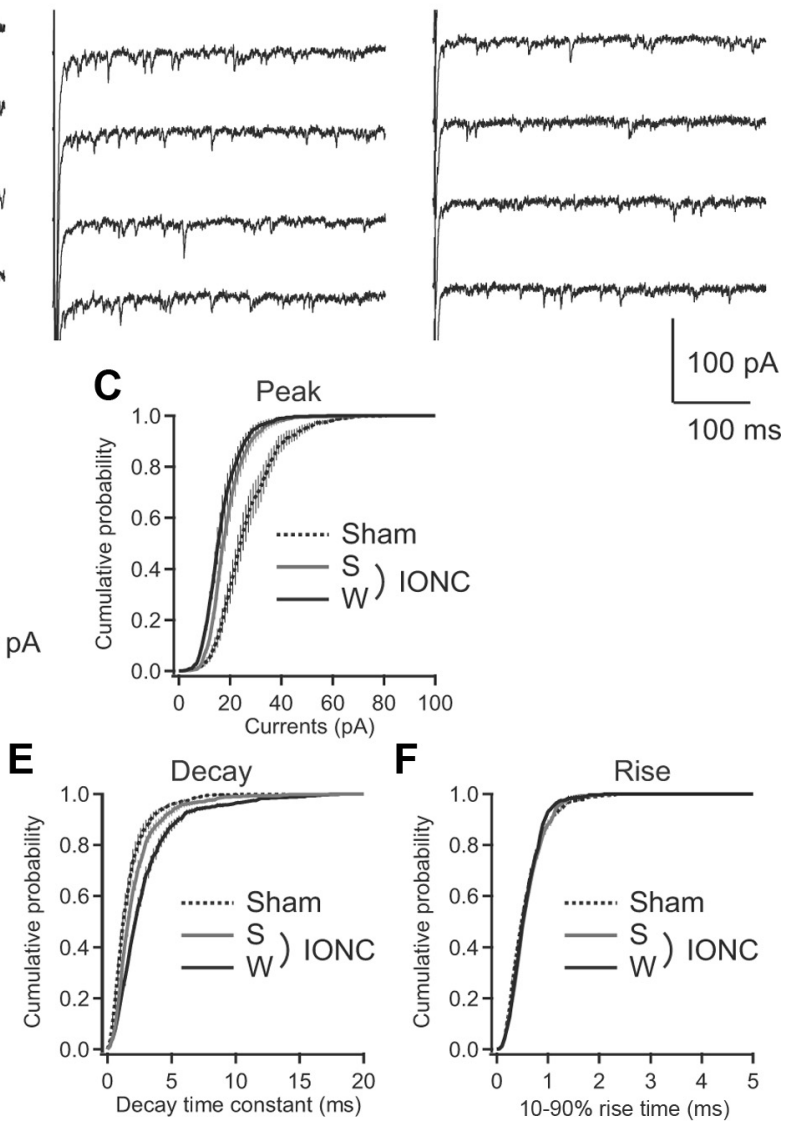

$5 \mathrm{~ms}$

$$
\text { Decay time constant (ms) }
$$

5

Figure 6. Analysis of asynchronous miniature EPSCs from individual lemniscal fibers. $A$, Representative traces of pure AMPARmeditated asynchronous miniature EPSCs evoked by single lemniscal fiber stimulation in the presence of $4 \mathrm{~mm} \mathrm{Sr}^{2+}$ ions. $\boldsymbol{B}$, 作 me data as in $\boldsymbol{B}$, but normalized by amplitude. $\boldsymbol{C}, \boldsymbol{E}, \boldsymbol{F}$, Mean cumulative probabilities of amplitude, decay time constants, and $10-90 \%$ rise time, respectively. Data are represented as the mean \pm SEM.

\section{IONC causes upregulation of GluA2 expression in the contralateral VPM}

To confirm the upregulation of GluA2 containing AMPARs after the IONC more directly, we used immunofluorescent labeling with antibodies against GluA2 and VGluT2, a marker for terminals in lemniscal fibers (Graziano et al., 2008) (Fig. 8). At lower magnification, fluorescence intensity for GluA2 in the VPM was much weaker than that in the cerebral cortex, the hippocampus, and the striatum (Fig. 8A). Nevertheless, signal intensity was higher in the contralateral VPM than in the ipsilateral VPM after the IONC (Fig. $8 A$ ). This difference was consistently observed in each of the three mice examined. At higher magnification, punctate immunolabeling for GluA2 was densely distributed in the neuropil, and some labeled puncta were associated with VGluT2positive terminals (Fig. $8 B, C$ ). Because VGluT2 in the VPM is only located at the terminals made by lemniscal fibers (Graziano et al., 2008), the fluorescence intensity of VGluT2-associated GluA2-positive puncta is a good index for quantifying the GluA2 expression level at lemniscal synapses. The fluorescence intensity of VGluT2-associated GluA2-positive puncta and the intensity in the adjacent GluA2-negative area were measured for GluA2 signal and background levels, respectively, in both the ipsilateral and contralateral VPM. The signal-to-background ratio in the contralateral VPM exhibited a significant increase $(\sim 40 \%)$ com- 
A

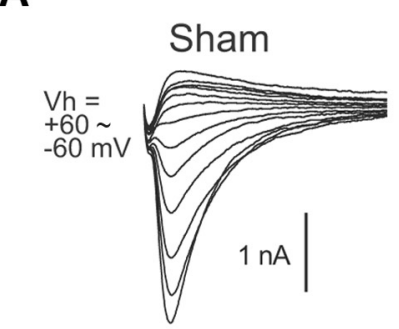

B

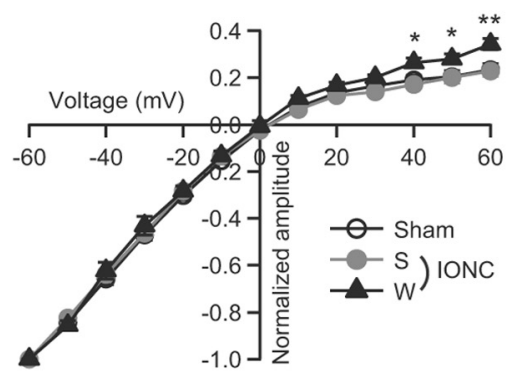

D
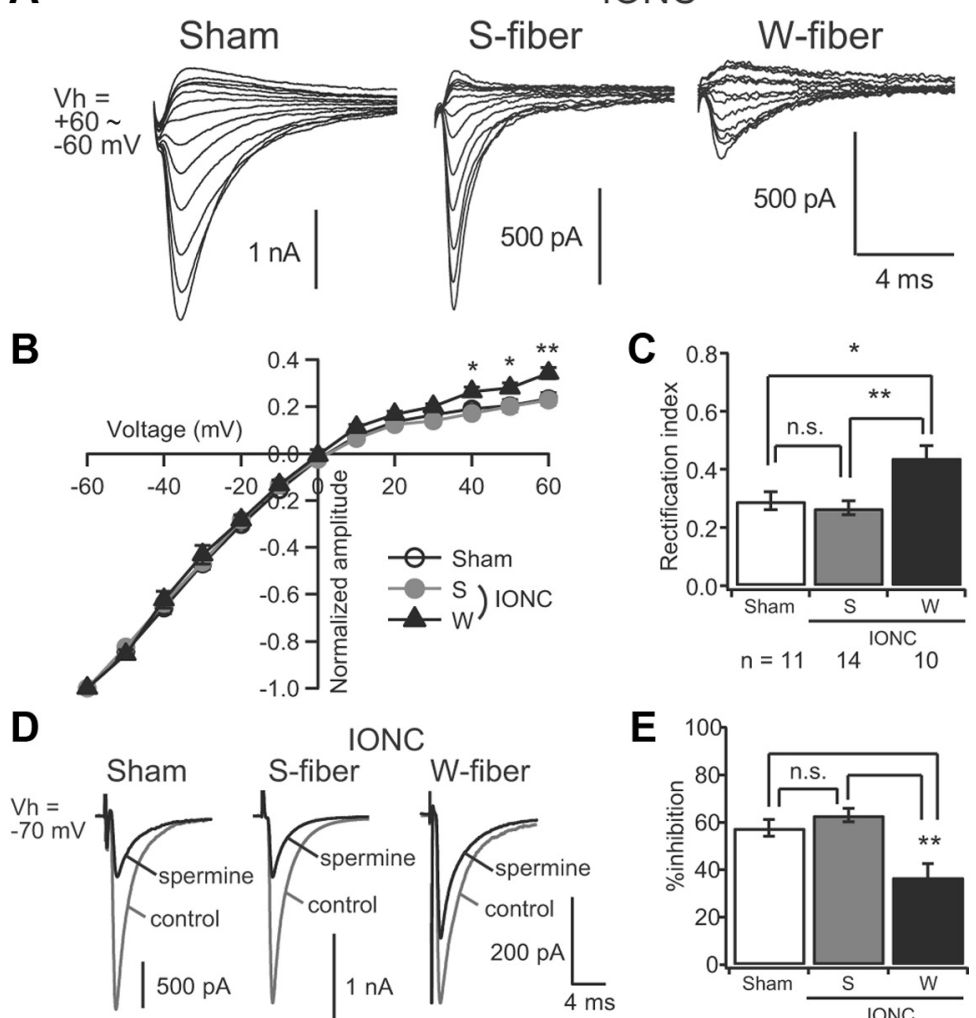

E

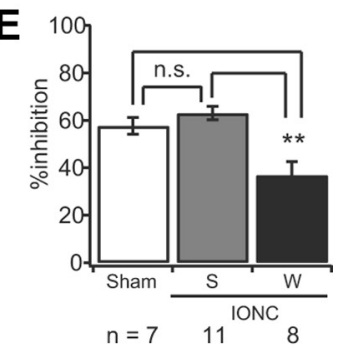

$\mathbf{F}$

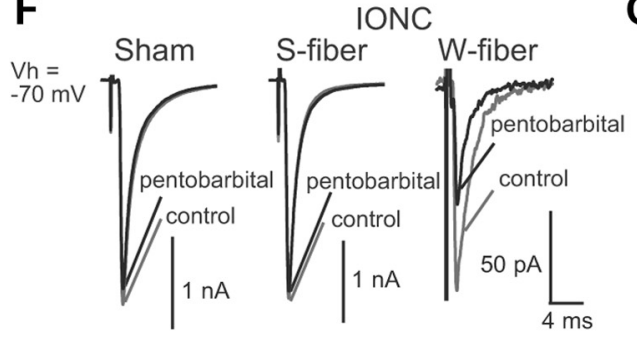

G

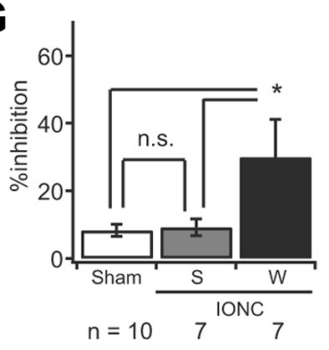

Figure 7. Relatively abundant GluA2-containing AMPARs at W-fiber synapses. $\boldsymbol{A}$, Representative raw traces of pure AMPARmediated lemniscal EPSCs at holding potentials ranged $\pm 60 \mathrm{mV}$ in $10 \mathrm{mV}$ steps in the three types of fibers. $\boldsymbol{B}$, Current-voltage relationships of pure AMPAR-mediated lemniscal EPSCs in the three types of fibers. Asterisks indicate a significant difference between sham fiber and W-fiber in the IONC group. C, Summary bar graphs showing rectification indexes defined as EPSC amplitude at $+40 \mathrm{mV}$ divided by that at $-40 \mathrm{mV}$. $n$, Number of fibers. $\boldsymbol{D}$, Representative traces of the AMPAR-mediated lemniscal EPSCs in the presence (black) and absence (gray) of exogenously applied $1 \mathrm{~mm}$ spermine, an inhibitor of GluA2-lacking AMPARs. Each trace is the average of six consecutive raw traces. $\boldsymbol{E}$, Summary bar graphs showing inhibitory effects of spermine on EPSC amplitude. $n$, Number of fibers. $\boldsymbol{F}, \boldsymbol{G}$, The same conventions as in $\boldsymbol{D}$ and $\boldsymbol{E}$, respectively, but with $100 \mu \mathrm{m}$ pentobarbital, an inhibitor of GluA2-containing AMPARs. All data are represented as the mean \pm SEM. Statistical significance was tested by multiple $t$ test with Bonferroni's correction following one-way ANOVA. ${ }^{*} p<0.05$; ${ }^{* *} p<0.01$; n.s., not significant (two-tailed test).

pared with that in the ipsilateral VPM (mean \pm SEM, contralateral VPM: $129.1 \pm 4.8 n=727$ puncta; ipsilateral VPM: $81.2 \pm 1.7, n=800$ puncta; $p<0.001$, Mann-Whitney $U$ test), thereby confirming that the IONC operation resulted in the upregulation of GluA2 expression in the lesioned VPM.

\section{NMDAR-mediated component of lemniscal EPSCs after the IONC}

We next examined postoperative changes in NMDAR-mediated EPSCs on POD7-POD11. Similar to AMPAR-mediated EPSCs, NMDAR-mediated EPSCs of W-fibers were also smaller than those in both sham fibers and S-fibers in the IONC group (sham fibers: $1.22 \pm 0.11 \mathrm{nA}, n=71$ fibers; S-fibers in the IONC group: $0.93 \pm 0.11 \mathrm{nA}, n=63$; and $\mathrm{W}$-fibers in the IONC group: $0.18 \pm$
$0.02 \mathrm{nA}, n=29$ fibers) (Fig. $9 A, B ;{ }^{* *} p<$ 0.01 , two-tailed multiple $t$ test with Bonferroni's correction following one-way ANOVA). However, the peak current ratio of NMDAR/AMPAR at W-fiber EPSCs was significantly larger than those at sham fiber EPSCs and S-fiber EPSCs in the IONC group (sham fibers: $0.42 \pm 0.03$, $n=53$ fibers; S-fibers in the IONC group: $0.45 \pm 0.03, n=60$ fibers; and $\mathrm{W}$-fibers in the IONC group: $0.90 \pm 0.06, n=29$ fibers) (Fig. $9 A, C ;{ }^{*} p<0.01$ ). On the other hand, no difference was observed in the $I-V$ relationship and decay time constant of NMDAR-mediated EPSCs $<20$ $\mu \mathrm{M}$ NBQX, a non-NMDA glutamate receptor antagonist, among the three fiber groups (Fig. 9D-G; both $p>0.05$ ). These results thus indicate that there was no change in the composition of NMDARs after the IONC.

\section{Altered presynaptic properties of W-fibers}

To assess presynaptic properties of the three types of fibers, paired-pulse ratio and coefficient of variation (CV) of EPSC amplitude were analyzed. The pairedpulse ratio (second EPSCs/first EPSCs) and $\mathrm{CV}$ value correlate negatively with the probability of transmitter release (Chuhma and Ohmori, 1998; Zucker and Regehr, 2002). The paired-pulse ratio of W-fiber EPSCs was larger than those of sham and $S$ fiber EPSCs, especially at short interstimulus intervals (Fig. $10 A, B ; p<$ 0.05 vs sham fiber; $p<0.01$ vs $S$-fiber, two-way repeated ANOVA; $n=6,10$, and 6 fibers for sham fiber, S-fiber, and $\mathrm{W}$-fiber in the IONC group, respectively). These results suggest that $\mathrm{W}$-fiber synapses have a lower release probability. Consistent with these results, the $\mathrm{CV}$ value (calculated from 30 consecutive EPSCs) of $\mathrm{W}$-fibers in the IONC group $(0.147 \pm 0.021)$ was significantly larger than that of sham fibers $(0.048 \pm 0.005)$ and S-fibers in the IONC group $(0.051 \pm$ 0.006 ) (Fig. $10 C,{ }^{* *} p<0.01,25$ fibers for both fiber types, two-tailed, multiple $t$ test with Bonferroni correction following one-way ANOVA). In contrast, both parameters were not different between S-fibers in the IONC group and sham fibers $(p>0.05)$.

Whisker deprivation and activity blockade of the infraorbital nerve do not induce new innervations by multiple lemniscal fibers

Previous studies showing that afferent fibers onto a thalamic relay neuron are rewired in the late absence of sensory experience in the mature visual thalamus (Hooks and Chen, 2006, 2008) raise the concern that IONC-induced innervations by multiple lemniscal fibers could be attributed to the lack of sensory experience. To test this possibility, we plucked out all large whiskers on the 

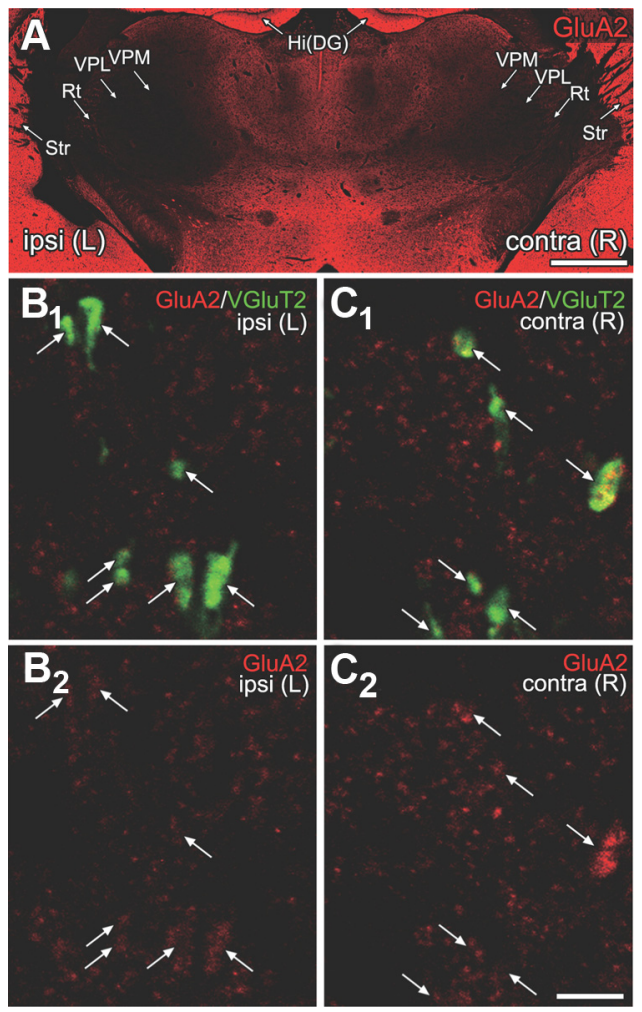

Figure 8. The IONC operation causes small but significant increase in GluA2 expression in the contralateral VPM. $A$, A photomontage at low magnification showing a mild increase in GluA2 immunoreactivity in the contralateral VPM. $\boldsymbol{B}, \boldsymbol{C}$, Images at high magnification showing double immunofluorescence for GluA2 (red) and VGluT2 (green) in the ipsilateral $\left(\boldsymbol{B}_{\boldsymbol{1}}\right)$ and contralateral $\left(\boldsymbol{C}_{\boldsymbol{1}}\right)$ VPM and single immunofluorescence for GluA2 ( $\boldsymbol{B}_{2}$, $\boldsymbol{C}_{2}$ ), respectively. Note that fluorescence intensity of VGluT2-associated GluA2 immunolabeling (indicated by arrows) is increased in the contralateral VPM. Hi (DG), Hippocampus (dentate gyrus); Rt, reticular nucleus of thalamus; Str, striatum; contra, contralateral; ipsi, ipsilateral. Scale bars: $\boldsymbol{A}, 1 \mathrm{~mm}$; (in $\boldsymbol{C}_{2}$ ) $\boldsymbol{B}$ and $\boldsymbol{C}, 5 \mu \mathrm{m}$.

left snout from P21 throughout recording days P28-P32 (Fig. $11 A$ ), the time course of which was identical to that of the IONC experiment. We found that there was no difference in the number of lemniscal fibers onto a relay neuron between the intact and deprived groups (Fig. $11 B, C ; p=1.00$, two-tailed KS test, $n=31$ and 24 cells for intact and deprived groups, respectively). Furthermore, in an additional set of experiments, we inhibited action potentials of the infraorbital nerve with TTX, which blocks both sensory inputs and spontaneous activity. We achieved continuous blockade of the activity of the infraorbital nerve by surgically implanting pieces of a slow-releasing polymer, EVAFLEX ${ }^{\mathrm{TM}}$, which contained TTX, on the infraorbital nerve from P21, then performing recordings on P28-P32 (Fig. 11D). The TTX treatment did not alter the number of lemniscal fiber inputs onto a relay neuron (Fig. $11 \mathrm{E}, F ; p=1.00$, two-tailed KS test, $n=6$ and 28 cells for saline- and TTX-treated groups, respectively). These results thus provide direct evidence that both sensory deprivation and activity blockade of the infraorbital nerve are not essential for the induction of IONC-mediated multiple innervation of lemniscal fibers onto a relay neuron.

\section{Discussion}

In the present study, we found a novel rewiring of lemniscal fibers onto a relay neuron in the contralateral VPM induced by the IONC operation (Fig. 1), which began around POD5POD6. The rewiring, by newly recruited multiple lemniscal fibers onto a single thalamic neuron, was pathway specific, but did not depend on the lack of sensory experience or the activity blockade of the ION. Furthermore, GluA2-containing AMPARs, which are negligible in the adult mouse VPM, were preferentially incorporated at newly formed lemniscal synapses.

\section{Newly recruited lemniscal fibers innervate a thalamic neuron} after the IONC from POD5 to POD6

It has been thought that the functional reorganization of receptive fields at supraspinal levels after peripheral sensory nerve injury occurs initially through changes in the strength and efficacy of existing synapses, rather than active structural alterations such as the sprouting of axons and the remodeling of neuronal circuits. Structural alterations at supraspinal levels are generally considered to take months or even years to occur after peripheral nerve injury, and this alternation is responsible for the consolidation of the reorganization of receptive fields over time after the injury (Kaas et al., 2008). Conversely, a recent study has revealed a rapid remodeling of spines in the somatosensory cortex after the injury of the sciatic nerve (Kim and Nabekura, 2011), although it still remains unclear whether the remodeling also takes place at the presynaptic axon terminals, which are a counterpart to the remodeled spines.

A remarkable finding in the present study is the rapid postoperative change in the rewiring of lemniscal synapses onto a single VPM neuron after the IONC. Our electrophysiological results demonstrate that $\mathrm{W}$-fibers, a type of newly recruited fiber, emerged around POD5-POD6. This time course of trans-synaptic rewiring, similar to that observed in the spinal cord and brainstem regions (Erzurumlu et al., 1989; Woolf et al., 1990, 1992), was faster than expected. Therefore, the rewiring of lemniscal fibers at the VPM proceeds in parallel to structural alterations at subthalamic levels after the IONC, long before the functional reorganization of receptive fields is consolidated (Pons et al., 1991; Jones and Pons, 1998).

After the IONC, the mean amplitude of single-fiber-mediated EPSCs was reduced, whereas those of maximal EPSCs on a relay neuron were maintained at the intact level (Fig. 3). These results suggest that the IONC-induced rewiring was in fact a form of homeostatic plasticity to maintain postsynaptic inputs onto a relay neuron; the reduced synaptic currents were compensated by recruiting additional lemniscal fibers. Decrease in the mean amplitude of AMPAR-mediated single-fiber lemniscal EPSCs in the IONC group was mainly due to the incorporation of W-fibers, because W-fiber EPSCs were evidently small. However, S-fiber synapses also became slightly weaker $(\sim 87.1 \%$ of sham fiber EPSCs) after the IONC. This may have stemmed from the decrease in mEPSC amplitude. There was no difference between the rise times of miniature and evoked EPSCs mediated by S- and Wfibers in the IONC group and sham fibers. In addition, the IONC did not influence the dendritic morphology, resting potential, membrane capacitance, or input resistance of relay neurons. Thus, newly recruited fibers presumably innervate soma or proximal dendrites of relay neurons as normal lemniscal fibers do (Peschanski et al., 1984). Although the source of additional recruited fibers is unclear, they may form new synapses adjacent to existing synapses, and/or replace synapses of previously connected lemniscal fibers, similar to those observed in neuromuscular junction after motor neuron injury (Brown et al., 1981). 
A
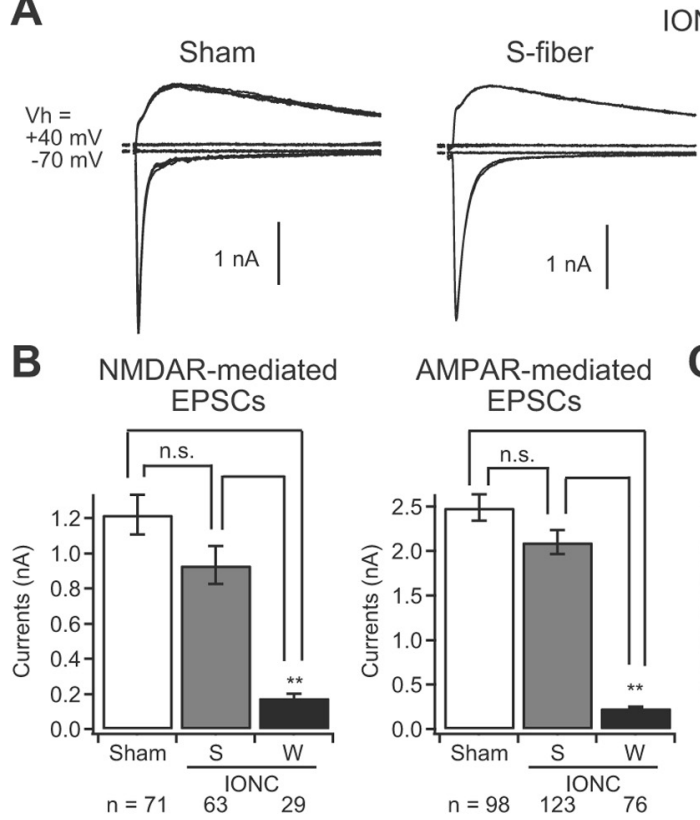

ONC

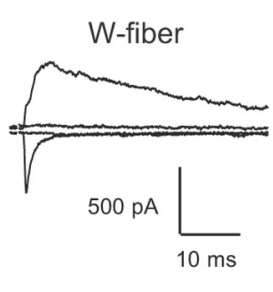

AMPAR-mediated
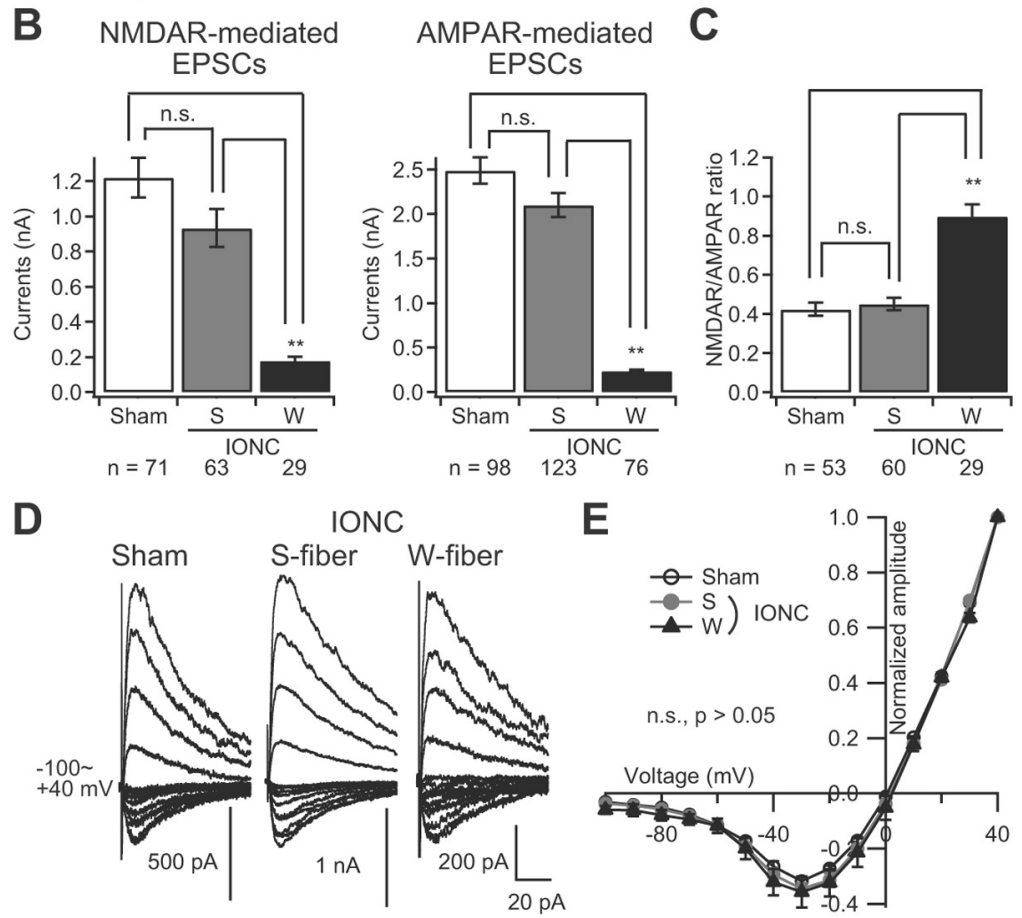

$\mathbf{F}$

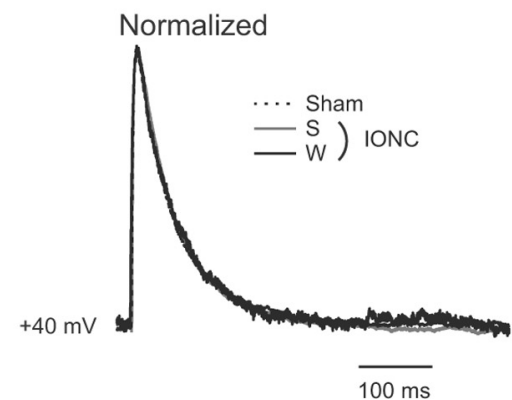

E

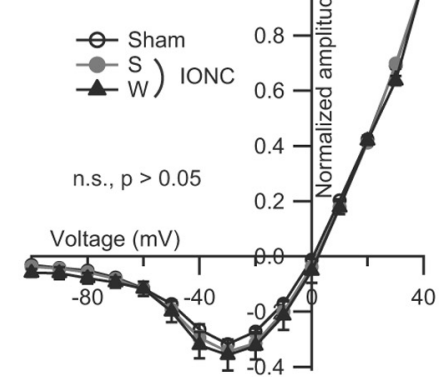

G

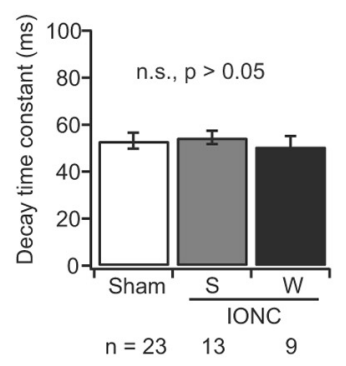

Figure 9. NMDAR-mediated component of lemniscal EPSCS after the IONC. $A$, Representative traces of lemniscal EPSCs mediated by a sham fiber, an S-fiber in the IONC group, and a W-fiber in the IONC group. Several raw traces with different stimulus intensities are superimposed. $\boldsymbol{B}$, Summary bar graphs showing the mean of amplitude of NMDAR-mediated (left) and AMPARmediated (right) EPSCs. $n$, number of fibers. C, Summary bar graph showing the mean of NMDAR/AMPAR ratios $(+40 /-70 \mathrm{mV})$ from single-fiber-mediated lemniscal EPSC $s$. n, number of fibers. $\boldsymbol{D}$, Representative raw traces of pure NMDAR-mediated lemniscal EPSCs at holding potentials between +40 and $-100 \mathrm{mV}$ in $10 \mathrm{mV}$ steps with $<20 \mu \mathrm{m} \mathrm{NBQX}$. E, Current-voltage relationships of pure NMDAR-mediated lemniscal EPSCS. The number of tested fibers was 9,10 , and 10, respectively, for sham fibers, $S$-fibers in the IONC group, and W-fibers in the IONC group. $\boldsymbol{F}$, Representative traces of pure NMDAR-mediated EPSCs are normalized by their peak amplitudes. Peak currents were 671, 1321, and 261 pA, respectively, for a sham fiber, an S-fiber in the IONC group, and a W-fiber in the IONC group. G, Summary bar graphs showing the decay time constants of NMDAR-mediated EPSCs from each of three kinds of fibers. $n$, Number of fibers. All data are expressed as the mean \pm SEM. Statistical significance was tested by two-tailed multiple $t$ test with Bonferroni's correction following one-way ANOVA $(\boldsymbol{B}, \boldsymbol{C})$, one-way ANOVA $(\boldsymbol{G})$, and two-way repeated ANOVA $(\boldsymbol{E})$, respectively. ${ }^{* *} p<0.01$. n.s., Not significant.

Relatively rich GluA2-containing AMPARs and distinct presynaptic properties at newly formed synapses

The rodent somatosensory thalamus in normal adults exhibits very little expression of GluA2 mRNA and GluA2 proteins (Sato et al., 1993; Mineff and Weinberg, 2000). In particular, matured lemniscal synaptic responses are mainly mediated by GluA2lacking AMPARs (Arsenault and Zhang, 2006; Wang and Zhang, 2008), which contribute most likely to the precisely timed firing of relay neurons in response to afferent inputs (Montemurro et al., 2007). In this context, it is of great interest to note in our study that the IONC upregulated GluA2-containing AMPARs selectively at newly recruited fiber synapses (W-fiber synapses). GluA2 has dominant roles in the AMPAR trafficking to synaptic sites through the interaction with receptor-trafficking proteins (Collingridge et al., 2004). Exocytosis of GluA2 is rapid and occurs constitutively under the basal condition, leading to stabilization of synaptic strength (Passafaro et al., 2001). Recently, it was also found that the N-terminal domain of GluA2 interacts with $\mathrm{N}$-cadherin, which activates cofilin-mediated signals, and this complex is associated with structural and functional synaptic plasticity (Silverman et al., 2007; Zhou et al., 2011). Thus, it is likely that GluA2 plays a crucial role in not only establishing the receptor number at synapses, but also the structural plasticity of spines. Furthermore, from a developmental perspective, the GluA2 subunit is highly expressed during the early developmental stage and declines gradually during maturation in the thalamus (Pellegrini-Giampietro et al., 1992; Spreafico et al., 1994). A large NMDAR/ AMPAR ratio, which was exhibited at $\mathrm{W}$-fiber synapses in our study, is frequently observed at immature synapses in several kinds of neurons. In addition, large paired-pulse ratio and CV values at $\mathrm{W}$-fiber synapses suggest the lower release probability, which is also reported at immature synapses (Chuhma and Ohmori, 1998). Therefore, W-fibers may have a feature of immature synapses. In contrast, S-fiber synapses were of the same nature as sham fiber synapses in the presynaptic properties and postsynaptic glutamate composition, strongly suggesting that $\mathrm{S} \mathrm{fi-}$ bers are pre-existent fibers.

\section{Sensory deprivation is not crucial for the IONC-induced rewiring of lemniscal fibers}

Our results indicate that the IONCmediated rewiring of lemniscal fibers was sensory pathway specific. Neither sensory deprivation nor the activity blockade of the ION was essential for the rewiring of lemniscal fibers. These results are consistent with evidence that axon branches are relatively stable under sensory deprivation in the somatosensory cortex of mature mice (Tra- 

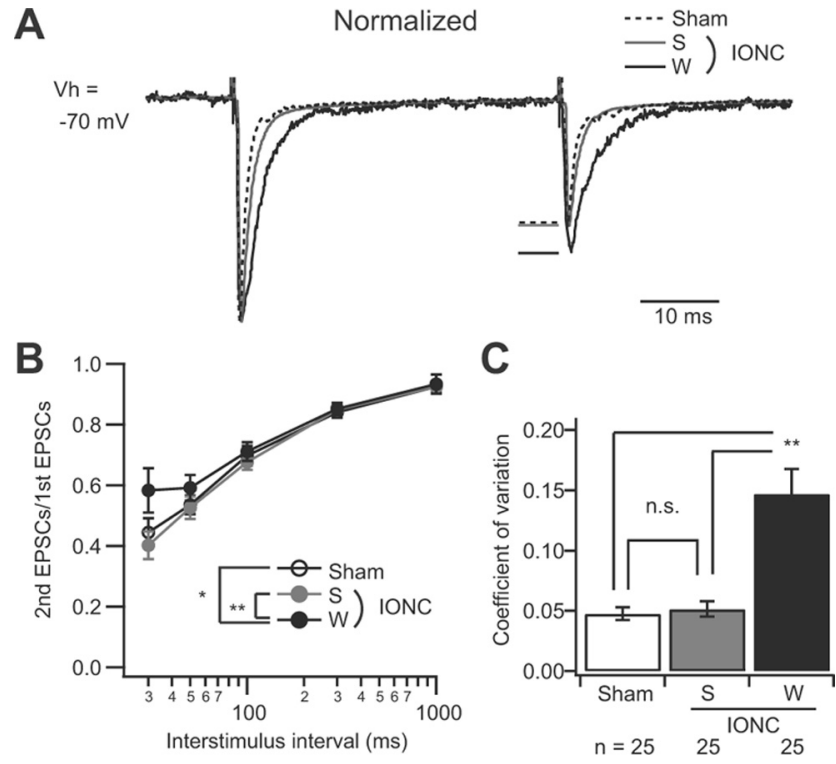

Figure 10. Altered presynaptic properties at W-fiber synapses. $\boldsymbol{A}$, Representative traces of lemniscal EPSCs evoked by paired-pulse stimulation at a $50 \mathrm{~ms}$ interstimulus interval. Traces were normalized by peak amplitude of the first EPSC $(2101,5829$, and 381 pA, respectively, for a sham fiber, an S-fiber in the IONC group, and a W-fiber in the IONC group). Horizontal lines indicate peak amplitudes of the second EPSCs. $\boldsymbol{B}$, The mean of paired-pulse ratios plotted as a function of the interstimulus interval. The numbers of tested fibers are 6, 10, and 6, respectively, for sham fibers, S-fibers in the IONC group, and W-fibers in the IONC group. Each point represents the mean \pm SEM. Statistical significance was tested by two-way repeated ANOVA. ${ }^{*} p<$ 0.05 . ${ }^{* *} p<0.01$. C, The coefficient of variation of 30 consecutive EPSC amplitudes was calculated and summarized. Each column represents the mean \pm SEM from 25 separate fibers. Statistical significance was tested by two-tailed multiple $t$ test with Bonferroni's correction following one-way ANOVA. ${ }^{* *} p<0.01$.

chtenberg et al., 2002). Moreover, the spontaneous firing rate in the rodent principle trigeminal nucleus is very low $(<1 \mathrm{~Hz})$ (Waite, 1984). In our preliminary experiment, the spontaneous firing of neurons in the nucleus did not differ between the sham and IONC groups in anesthetized mice. Thus, the activity of the trigeminal nucleus may not also be essential. In contrast to the somatosensory pathway, the rewiring of retinogeniculate fibers on a thalamic relay neuron depends largely on late visual experience in mature mice (Hooks and Chen, 2006, 2008). These results suggest that the triggers to the rewiring of afferent fibers in the thalamus are different in each sensory pathway.

Other than the activity dependence, there may be other mechanisms underlying the rewiring of lemniscal fibers. It has been previously reported that BDNF can promote the survival of specific neuronal populations and growth of their neurites through TrkB after peripheral nerve injury (Boyd and Gordon, 2003). A recent study has demonstrated that the upregulation of BDNF in microglia also plays an important role in the axonal regeneration of injured primary afferents in the mesencephalic trigeminal tract nucleus of the rodent (Ichikawa et al., 2011). Thus, upregulation of BDNF by microglial activation in the trigeminal nucleus may underlie the rewiring of lemniscal fibers. Another possibility is that the axonal transport from peripheral to the trigeminal nucleus may be disrupted by the IONC. Previous studies have revealed that the axonal transport, but not nerve activity, is required for proper neural circuitry during development (Henderson et al., 1992; Chiaia et al., 1996). The axonal transport may also play a prominent role in maintaining the proper neural wiring even in the adult circuitry.
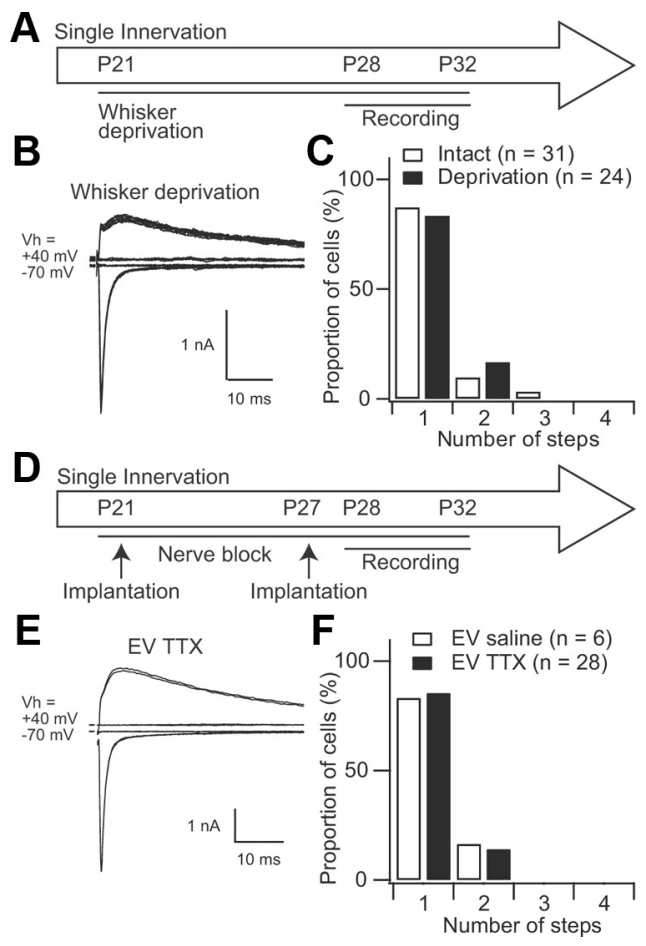

Figure 11. Neither whisker deprivation nor activity blockade of the infraorbital nerve induces multiple innervation of lemniscal fibers. $A$, The experimental schedule of whisker deprivation, which was performed every day from P21 to the recording day. $\boldsymbol{B}$, Representative traces of lemniscal EPSCs from a deprived mouse, showing an all-or-none fashion. C, The distributions of neurons with different numbers of lemniscal inputs for the intact and deprived VPM. $n$, Number of cells. $\boldsymbol{D}$, The experimental schedule of the infraorbital nerve blockade. Each arrow indicates the implantation day of EVAFLEXTM (EV) pieces containing either saline or tetrodotoxin (TTX). E, Representative traces of lemniscal EPSCs after EV-TTX implantation, showing an allor-none response. $\boldsymbol{F}$, The distributions of relay neurons in the VPM with different numbers of lemniscal inputs for the EV-saline-treated and EV-TTX-treated mice. $n$, Number of cells.

\section{Functional implication of the IONC-induced rewiring of lemniscal fibers}

It is generally believed that there are three phases in the reorganization process after peripheral nerve injury (Wall et al., 2002): an immediate phase of changes in the receptive fields by unmasking pre-existing neural circuitry that is normally latent; a subacute phase lasting weeks or months, during which the new representation of the receptive fields is consolidated, but its topographic order is restored; and a late phase for consolidation of the final reorganization of receptive fields, such as the usedependent refinement of the internal topography. The IONCinduced rewiring of lemniscal fibers occurs in the subacute phase after peripheral nerve injury. Newly recruited lemniscal fibers (W-fibers) may participate in the expansion of representations and/or the emergence of the new representation of receptive fields, which are observed in the subacute phase. Although a Wfiber has $<500 \mathrm{pA}$ of an AMPAR-mediated EPSC, the specific expression of a GluA2-containing AMPAR and a relatively rich NMDAR, both of which have slow kinetics, ensure larger synaptic charge. Therefore, a W-fiber may drive the relay neuron firing as an immature retinogeniculate fiber does (Liu and Chen, 2008). In addition, these postsynaptic features of $\mathrm{W}$-fiber synapses may underlie abnormal firing in thalamic relay neurons after peripheral nerve injury (Lenz et al., 1998). Future studies are warranted to elucidate the consequence of the multiple innervation of lemniscal fibers over time; that is, how the rewiring process eventu- 
ally refines and consolidates the reorganized somatosensory thalamic circuitry in response to the injury.

\section{References}

Arsenault D, Zhang ZW (2006) Developmental remodelling of the lemniscal synapse in the ventral basal thalamus of the mouse. J Physiol 573:121-132.

Bowie D, Mayer ML (1995) Inward rectification of both AMPA and kainate subtype glutamate receptors generated by polyamine-mediated ion channel block. Neuron 15:453-462.

Boyd JG, Gordon T (2003) Neurotrophic factors and their receptors in axonal regeneration and functional recovery after peripheral nerve injury. Mol Neurobiol 27:277-324.

Brown MC, Holland RL, Hopkins WG (1981) Motor nerve sprouting. Annu Rev Neurosci 4:17-42.

Castro-Alamancos MA (2002) Properties of primary sensory (lemniscal) synapses in the ventrobasal thalamus and the relay of high-frequency sensory inputs. J Neurophysiol 87:946-953.

Chiaia NL, Bennett-Clarke CA, Crissman RS, Zheng L, Chen M, Rhoades RW (1996) Effect of neonatal axoplasmic transport attenuation in the infraorbital nerve on vibrissae-related patterns in the rat's brainstem, thalamus and cortex. Eur J Neurosci 8:1601-1612.

Chuhma N, Ohmori H (1998) Postnatal development of phase-locked high-fidelity synaptic transmission in the medial nucleus of the trapezoid body of the rat. J Neurosci 18:512-520.

Collingridge GL, Isaac JT, Wang YT (2004) Receptor trafficking and synaptic plasticity. Nat Rev Neurosci 5:952-962.

Dunwiddie T, Lynch G (1978) Long-term potentiation and depression of synaptic responses in the rat hippocampus: localization and frequency dependency. J Physiol 276:353-367.

Erzurumlu RS, Jhaveri S, Moya KL, Benowitz LI (1989) Peripheral nerve regeneration induces elevated expression of GAP-43 in the brainstem trigeminal complex of adult hamsters. Brain Res 498:135-139.

Flor H, Nikolajsen L, Staehelin Jensen T (2006) Phantom limb pain: a case of maladaptive CNS plasticity? Nat Rev Neurosci 7:873-881.

Florence SL, Taub HB, Kaas JH (1998) Large-scale sprouting of cortical connections after peripheral injury in adult macaque monkeys. Science 282:1117-1121.

Fraley C, Raftery AE (2002) Model-based clustering, discriminant analysis, and density estimation. J Am Stat Assoc 97:611-631.

Fuchs EC, Doheny H, Faulkner H, Caputi A, Traub RD, Bibbig A, Kopell N, Whittington MA, Monyer H (2001) Genetically altered AMPA-type glutamate receptor kinetics in interneurons disrupt long-range synchrony of gamma oscillation. Proc Natl Acad Sci U S A 98:3571-3576.

Geiger JR, Melcher T, Koh DS, Sakmann B, Seeburg PH, Jonas P, Monyer H (1995) Relative abundance of subunit mRNAs determines gating and $\mathrm{Ca}^{2+}$ permeability of AMPA receptors in principal neurons and interneurons in rat CNS. Neuron 15:193-204.

Graziano A, Jones EG (2009) Early withdrawal of axons from higher centers in response to peripheral somatosensory denervation. J Neurosci 29:3738-3748.

Graziano A, Liu XB, Murray KD, Jones EG (2008) Vesicular glutamate transporters define two sets of glutamatergic afferents to the somatosensory thalamus and two thalamocortical projections in the mouse. J Comp Neurol 507:1258-1276.

Henderson TA, Woolsey TA, Jacquin MF (1992) Infraorbital nerve blockade from birth does not disrupt central trigeminal pattern formation in the rat. Brain Res Dev Brain Res 66:146-152.

Hooks BM, Chen C (2006) Distinct roles for spontaneous and visual activity in remodeling of the retinogeniculate synapse. Neuron 52:281-291.

Hooks BM, Chen C (2008) Vision triggers an experience-dependent sensitive period at the retinogeniculate synapse. J Neurosci 28:4807-4817.

Ichikawa H, Sato T, Kano M, Suzuki T, Matsuo S, Kanetaka H, Shimizu Y (2011) Masseteric nerve injury increases expression of brain-derived neurotrophic factor in microglia within the rat mesencephalic trigeminal tract nucleus. Cell Mol Neurobiol 31:551-559.

Isa T, Iino M, Itazawa S, Ozawa S (1995) Spermine mediates inward rectification of $\mathrm{Ca}^{2+}$-permeable AMPA receptor channels. Neuroreport 6:2045-2048.

Isa T, Iino M, Ozawa S (1996) Spermine blocks synaptic transmission mediated by $\mathrm{Ca}^{2+}$-permeable AMPA receptors. Neuroreport 7:689-692.
Jones EG, Pons TP (1998) Thalamic and brainstem contributions to largescale plasticity of primate somatosensory cortex. Science 282:1121-1125.

Kaas JH, Qi HX, Burish MJ, Gharbawie OA, Onifer SM, Massey JM (2008) Cortical and subcortical plasticity in the brains of humans, primates, and rats after damage to sensory afferents in the dorsal columns of the spinal cord. Exp Neurol 209:407-416.

Kakizawa S, Miyazaki T, Yanagihara D, Iino M, Watanabe M, Kano M (2005) Maintenance of presynaptic function by AMPA receptor-mediated excitatory postsynaptic activity in adult brain. Proc Natl Acad Sci U S A 102:19180-19185.

Kim SK, Nabekura J (2011) Rapid synaptic remodeling in the adult somatosensory cortex following peripheral nerve injury and its association with neuropathic pain. J Neurosci 31:5477-5482.

Koerber HR, Mirnics K, Brown PB, Mendell LM (1994) Central sprouting and functional plasticity of regenerated primary afferents. J Neurosci 14:3655-3671.

Lenz FA, Garonzik IM, Zirh TA, Dougherty PM (1998) Neuronal activity in the region of the thalamic principal sensory nucleus (ventralis caudalis) in patients with pain following amputations. Neuroscience 86:1065-1081.

Li X, Glazewski S, Lin X, Elde R, Fox K (1995) Effect of vibrissae deprivation on follicle innervation, neuropeptide synthesis in the trigeminal ganglion, and S1 barrel cortex plasticity. J Comp Neurol 357:465-481.

Liu SJ, Zukin RS (2007) $\mathrm{Ca}^{2+}$-permeable AMPA receptors in synaptic plasticity and neuronal death. Trends Neurosci 30:126-134.

Liu X, Chen C (2008) Different roles for AMPA and NMDA receptors in transmission at the immature retinogeniculate synapse. J Neurophysiol 99:629-643.

McLachlan GJ, Chang SU (2004) Mixture modelling for cluster analysis. Stat Methods Med Res 13:347-361.

Mineff EM, Weinberg RJ (2000) Differential synaptic distribution of AMPA receptor subunits in the ventral posterior and reticular thalamic nuclei of the rat. Neuroscience 101:969-982.

Miyata M, Imoto K (2006) Different composition of glutamate receptors in corticothalamic and lemniscal synaptic responses and their roles in the firing responses of ventrobasal thalamic neurons in juvenile mice. J Physiol 575:161-174.

Miyazaki T, Fukaya M, Shimizu H, Watanabe M (2003) Subtype switching of vesicular glutamate transporters at parallel fibre-Purkinje cell synapses in developing mouse cerebellum. Eur J Neurosci 17:2563-2572.

Montemurro MA, Panzeri S, Maravall M, Alenda A, Bale MR, Brambilla M, Petersen RS (2007) Role of precise spike timing in coding of dynamic vibrissa stimuli in somatosensory thalamus. J Neurophysiol 98:1871-1882.

Mosbacher J, Schoepfer R, Monyer H, Burnashev N, Seeburg PH, Ruppersberg JP (1994) A molecular determinant for submillisecond desensitization in glutamate receptors. Science 266:1059-1062.

Passafaro M, Piëch V, Sheng M (2001) Subunit-specific temporal and spatial patterns of AMPA receptor exocytosis in hippocampal neurons. Nat Neurosci 4:917-926.

Pellegrini-Giampietro DE, Bennett MV, Zukin RS (1992) Are $\mathrm{Ca}^{2+}$ permeable kainate/AMPA receptors more abundant in immature brain? Neurosci Lett 144:65-69.

Peschanski M, Lee CL, Ralston HJ 3rd (1984) The structural organization of the ventrobasal complex of the rat as revealed by the analysis of physiologically characterized neurons injected intracellularly with horseradish peroxidase. Brain Res 297:63-74.

Pons TP, Garraghty PE, Ommaya AK, Kaas JH, Taub E, Mishkin M (1991) Massive cortical reorganization after sensory deafferentation in adult macaques. Science 252:1857-1860.

Sato K, Kiyama H, Tohyama M (1993) The differential expression patterns of messenger RNAs encoding non- $N$-methyl-D-aspartate glutamate receptor subunits (GluR1-4) in the rat brain. Neuroscience 52:515-539.

Silverman JB, Restituito S, Lu W, Lee-Edwards L, Khatri L, Ziff EB (2007) Synaptic anchorage of AMPA receptors by cadherins through neural plakophilin-related arm protein AMPA receptor-binding protein complexes. J Neurosci 27:8505-8516.

Spreafico R, Frassoni C, Arcelli P, Battaglia G, Wenthold RJ, De Biasi S (1994) Distribution of AMPA selective glutamate receptors in the thalamus of adult rats and during postnatal development. A light and ultrastructural immunocytochemical study. Brain Res Dev Brain Res $82: 231-244$.

Trachtenberg JT, Chen BE, Knott GW, Feng G, Sanes JR, Welker E, Svoboda 
$\mathrm{K}$ (2002) Long-term in vivo imaging of experience-dependent synaptic plasticity in adult cortex. Nature 420:788-794.

Waite PM (1984) Rearrangement of neuronal responses in the trigeminal system of the rat following peripheral nerve section. J Physiol 352:425-445.

Wall JT, Xu J, Wang X (2002) Human brain plasticity: an emerging view of the multiple substrates and mechanisms that cause cortical changes and related sensory dysfunctions after injuries of sensory inputs from the body. Brain research Brain research reviews 39:181-215.

Wang H, Zhang ZW (2008) A critical window for experience-dependent plasticity at whisker sensory relay synapse in the thalamus. J Neurosci 28:13621-13628.

Washburn MS, Dingledine R (1996) Block of $\alpha$-amino-3-hydroxy-5methyl-4-isoxazolepropionic acid (AMPA) receptors by polyamines and polyamine toxins. J Pharmacol Exp Ther 278:669-678.

Wiser AK, Callaway EM (1996) Contributions of individual layer 6 pyramidal neurons to local circuitry in macaque primary visual cortex. J Neurosci $16: 2724-2739$.

Woolf CJ, Reynolds ML, Molander C, O’Brien C, Lindsay RM, Benowitz LI
(1990) The growth-associated protein GAP-43 appears in dorsal root ganglion cells and in the dorsal horn of the rat spinal cord following peripheral nerve injury. Neuroscience 34:465-478.

Woolf CJ, Shortland P, Coggeshall RE (1992) Peripheral nerve injury triggers central sprouting of myelinated afferents. Nature 355:75-78.

Yamakura T, Sakimura K, Mishina M, Shimoji K (1995) The sensitivity of AMPA-selective glutamate receptor channels to pentobarbital is determined by a single amino acid residue of the $\alpha 2$ subunit. FEBS Lett 374:412-414.

Yamazaki M, Fukaya M, Hashimoto K, Yamasaki M, Tsujita M, Itakura M, Abe M, Natsume R, Takahashi M, Kano M, Sakimura K, Watanabe M (2010) TARPs $\gamma-2$ and $\gamma-7$ are essential for AMPA receptor expression in the cerebellum. Eur J Neurosci 31:2204-2220.

Zhou Z, Hu J, Passafaro M, Xie W, Jia Z (2011) GluA2 (GluR2) regulates metabotropic glutamate receptor-dependent long-term depression through $\mathrm{N}$-cadherin-dependent and cofilin-mediated actin reorganization. J Neurosci 31:819-833.

Zucker RS, Regehr WG (2002) Short-term synaptic plasticity. Annu Rev Physiol 64:355-405. 